\title{
23. PETROLOGY, MINERALOGY, AND GEOCHEMISTRY OF THE FALKLAND PLATEAU BASEMENT ROCKS, SITE 330, DEEP SEA DRILLING PROJECT
}

\author{
John Tarney, Department of Geological Sciences, The University, Birmingham, United Kingdom
}

\begin{abstract}
The basement rocks sampled beneath Jurassic sediments in Cores 16 and 17 , Site 330, record a long and complex geological history. The oldest rocks present are a series of garnetiferous biotitic gneisses. Petrological and geochemical evidence suggest that these gneisses were mature pelitic, psammitic, and perhaps even arkosic sediments initially, but have suffered prolonged metamorphism and deformation. Thin pegmatic $\left(\pi_{1}\right)$ veins were intruded before the main deformation and the metamorphic climax. Emplacement of a later $\left(\pi_{2}\right)$ granite pegmatite and related veins was followed by considerable hydrothermal retrogression of the gneisses: replacement of early garnets by chlorite and brown biotites by green to colorless hydrobiotites. A second-generation garnet seems to have developed synchronously with the pegmatite intrusion. The plutonic history was completed by the intrusion of thin basic veins and a microsyenite, but there was later tectonic activity in the form of minor faulting and brecciation.

Uplift and erosion exposed the rocks as an undulating Jurassic land surface. This period was characterized by the development of a bedrock calcrete (caliche) in the subaerial zone and replacement of plagioclase by calcite at deeper levels in the core. Burial beneath Jurassic lagoonal sediments preserved the relationships.
\end{abstract}

\section{INTRODUCTION}

Site 330 was located on the eastern end of the Falkland Plateau at $50^{\circ} 55.19^{\prime} \mathrm{S}, 46^{\circ} 53.00^{\prime} \mathrm{W}$. Basement was encountered at 556 meters subbottom depth in Core Catcher 15 and Cores 16 and 17 beneath Jurassic sediments. An additional three pieces of basement (labeled "17, CC") were recovered from the bit following abandonment of Hole 330A. These have been grouped with the main core samples for the purposes of description. Altogether 7.3 meters of basement was recovered out of 19.5 meters cored. The low recovery might be accounted for by the fragmentation of the more friable micaceous rock types during drilling. A fairly unconsolidated quartz-rich sand, with abundant tourmaline and pyrite, was found immediately overlying the basement.

The main rock types recovered may be grouped as follows:

1) Garnetiferous biotitic gneisses with granular texture and weak to strong foliation and varying from biotite-rich to quartz-rich in composition.

2) Deformed $\mathrm{K}$-feldspar-rich garnet-bearing pegmatitic veins roughly conformable with the gneisses ( $\pi$ 1 pegmatites).

3) Coarse-grained $\mathrm{K}$-feldspar granite pegmatite $\left(\pi_{2}\right)$ with related thin pegmatitic veins emplaced more or less conformably into the gneisses.

4) Quartz veins, again conformable to the gneiss foliation.
5) Thin basic veins which cross-cut the gneiss foliation.

6) A microsyenite intrusion, chilled against the granite pegmatite.

7) Calcite-impregnated gneisses in the upper part of the core, representing what is probably a bedrock palaeocalcrete (caliche) formation.

The gneisses form the bulk of the cored basement and have undergone quite a complex metamorphic history. Petrological and geochemical evidence, outlined below, suggests they may be of sedimentary parentage.

For convenience, discussion of the effects of basement calcrete is treated separately from the earlier metamorphic and igneous history of the basement rocks.

\section{PETROLOGY OF THE BASEMENT GNEISSES}

\section{Metasedimentary Gneisses}

Rocks in this section include those with a dominant metamorphic fabric and which therefore represent the earliest members of the complex. Although few of the rocks are coarse grained and most are micaceous to a variable extent (including almost pure biotite) the term "gneiss" is used in preference to "schist" because in general quartz and feldspar rather than mica dominate the fabric. There are indications too (see below) that some of the rocks may have been much coarser grained, 
but have been recrystallized to give the present granular fabric.

Approximate modal compositions for some of the rock types examined are listed in Table 1. Quartz is the dominant mineral, forming $40 \%$ or more of the mode in most rocks. This high percentage is strongly indicative of a sedimentary parentage for most of the sequence. Rocks richer in biotite probably represent pelitic compositions, but most correspond to semipelite or quartzrich semipelite. A fourth type, rich in $\mathrm{K}$-feldspar and quartz, and generally rather coarser grained and pinkgray in color, could well represent an early pegmatitic fraction deformed along with the metasedimentary rocks during the recrystallization which produced the granular texture. Minor components of the gneisses include quartz veins (Plate 1, Figure 4) and narrow veins of $\mathrm{K}$-feldspar, both conformable and cross-cutting, and of course the later fractures filled with calcite, finegrained chlorite, sericite, pyrophyllite, or other finegrained indeterminate microcrystalline material.

The quartzitic gneisses generally have both biotite and plagioclase as additional components, but the proportions of the three minerals vary quite widely. Some lithological bands are obviously richer in $\mathrm{K}$ feldspar, though not obviously of pegmatitic origin; such bands may represent original arkose. Garnet is a common minor component in almost all rocks, but is not always obvious in hand specimen owing to its replacement by fine-grained almost isotropic pale green chlorite. There appear to be two generations of garnet present. The earlier, scattered throughout most gneisses in the core, forms small rounded grains of reddish- brown color. It is this type which is invariably replaced to some extent by chlorite (Plate 3, Figure 6). The later generation of garnet is red-purple in color, forms large irregular-shaped grains often intergrown with quartz in vermicular fashion, and is surrounded by quartz (Plate 4 , Figure 1). This garnet shows little or no alteration and is found also in the margin of the later coarse pegmatitic granite. Most likely this garnet formed at the time of emplacement of the granite pegmatite; the absence of plagioclase in its vicinity suggests that it may have formed at the expense of plagioclase. The smaller, rounded shape of the reddish-brown garnets, on the other hand, indicates that they grew during or before the development of the granular fabric.

There are two distinct types of biotite present. An intense reddish-brown pleochroic variety is variably replaced by a green pleochroic variety. Only where completely enclosed in quartz (in small rounded grains) is the primary variety preserved intact. The replacement process appears to involve exsolution of titanomagnetite and quartz parallel to the biotite plates (Plate 3, Figures 1, 2). The green variety shows further replacement by a progressively paler green biotite/chlorite, but even at advanced stages of degradation (in the late sericite/pyrophyllite veins) the chlorite is still optically length slow.

The plagioclase composition is in the range $A_{25-35}$ in many of the granular quartz gneisses-a surprisingly calcic composition for such quartz-rich rocks. It is perhaps for this reason that the plagioclase is frequently badly clouded and partly replaced by calcite or siderite throughout most of the section (Plate 4, Figure 5). The

TABLE 1

Modal Mineral Proportions of Basement Rocks (Estimated \%)

\begin{tabular}{l|l|l|llll}
\hline & & & & \\
& & & &
\end{tabular}


more sodic plagioclase $\left(A_{20}\right)$ in the $\mathrm{K}$-feldspar-rich gneisses is less clouded and less susceptible to replacement by calcite. It may be worth nothing at this point that acid gneisses in high-grade granulite facies terrains have rather calcic compositions in the $\mathrm{An}_{30-40}$ range (Sheraton et al., 1973).

The potassic feldspar in the gneisses is also rather clouded. It is also generally untwinned and has rather low birefringence, and is uniaxially negative, or perhaps in the majority of cases biaxially negative, but with a very small $2 \mathrm{~V}$. Only in the $\mathrm{K}$-feldspar-rich (pegmatitic) granular gneisses is there a development of incipient cross-hatched twinning characteristic of microcline. Associated with this is an increase in the $2 \mathrm{~V}$ angle.

Of the minor components, tourmaline is the most noteworthy. It occurs in some of the more foliated garnetiferous biotite-rich gneisses and is elongated (10:1) parallel to the foliation (Plate 3, Figure 5). Tourmaline is a prominent component of the sandy soil (Core 15) overlying the basement gneisses. Small elongated granules of what (optically) appears to be topaz were located in Sample 16-2, $47 \mathrm{~cm}$. Zircon and apatite are locally concentrated in the rocks, but there is little primary ore.

The pelitic gneisses (e.g., Sample 16-2, $9 \mathrm{~cm}$ ) appear in hand specimen to be composed entirely of biotite (Plate 1, Figure 2; Plate 3, Figure 1). Thin-section examination reveals them to have been garnetiferous, although most of the garnet is replaced by siderite and fine-grained, almost isotropic chlorite.

The deformed pegmatitic gneisses (e.g., Sample 16-2, $47 \mathrm{~cm}$ ) are rich in K-feldspar and quartz, but poor in plagioclase and biotite (Plate 1, Figure 1; Plate 2, Figure 1). They are also garnetiferous, the early generation garnet being partly replaced by fine grained chlorite (Plate 3, Figure 5). Rounded chlorite clots (after garnet) in other pegmatitic lenses serve to distinguish early pegmatites $\left(\pi_{1}\right)$ from late pegmatites $\left(\pi_{2}\right)$.

\section{Fabric of the Gneisses}

The texture of the gneisses is dominantly granular (Plate 1, Figure 1). There is a foliation and a lithological banding obvious in hand specimen, but this is variable. This foliation is inclined at about $30^{\circ}$ relative to the core barrel (Plate 1, Figures 1-4). In some parts of the core there is no obvious lithological banding over distances as great as $20 \mathrm{~cm}$. Elsewhere both a lithological banding and a foliation marked by variations in proportion of biotite are obvious. Splitting of parts of the core showed, however, that much of the lithological banding is discontinuous and lensoid. The biotite-rich horizons are much more persistent.

Thin sections revealed that most of the rocks have a granular to subgranular texture (Plate 3, Figure 4; Plate 4 , Figures 3,4). Quartzes and feldspars have a grain size approximating $1 \mathrm{~mm}$, but are only slightly elongated parallel to the foliation. Most of the foliation is in fact defined by biotite (Plate 1, Figure 2). The biotite is invariably an aggregate of individual multicolored laths. But while the laths themselves are aligned parallel to the foliation, the form of the aggregates particularly in quartz-rich rocks is more equant and of a shape determined by the form of the surrounding quartzes and feldspars. The biotite fabric is of course better defined in the more biotite-rich rocks, where the quartzes and feldspars are also slightly more elongated.

Closer observation of the fabric of the quartzes revealed two other facts. First, that many adjacent individual granular quartz grains are optically continuous and are obviously part of originally single much larger quartz grains which have been recrystallized. These larger "composite" grains tend to be elongated roughly perpendicular to the present biotite foliation (Plate 3, Figure 4). Second, many of the granulites show prominent ore-trails in the quartzes which are surprisingly consistent and parallel. In appropriately oriented sections, these are also aligned roughly perpendicular to the present foliation.

Individually, each observation noted above is probably of limited significance, but taken as a whole they suggest that the present even-grained granular texture of these rocks may have been derived by recrystallization of some previous fabric in a much coarser grained rock type. It is possible also that the biotites have replaced some more equant mineral such as hypersthene by a reaction such as

$$
3 \mathrm{Hyp}+\mathrm{Or}+\text { water } \rightarrow \text { biotite }+\mathrm{SiO}_{2}
$$

although there is no evidence of remnant hypersthene in thin section.

\section{Granite Pegmatite}

Almost 1.4 meters of coarse-grained granitepegmatite was cored in the lower section of Core 17 before the hole was terminated. The lower contact of the pegmatite is set against the fine-grained margin of the late syenite intrusion, while the rather diffuse upper contact with the granulitic gneisses is preserved in the upper part of Core 17, Section 3.

The pegmatite is somewhat variable in texture and mineralogy, as is typical of such rocks. The upper contact with the gneisses has a grain size of less than $0.5 \mathrm{~cm}$ and is distinctly richer in plagioclase and quartz relative to the center. The central part of the pegmatite, as cored, has a grain size of approzimately $1 \mathrm{~cm}$ (Plate 2, Figure 2), but this zone is flanked on either side by zones with potash feldspars several centimeters in diameter, intergrown with quartz and biotite. This heterogeneity precludes an accurate assessment of mineral proportions, but these could be estimated as $\mathrm{K}$ feldspar $65 \%$, quartz $20 \%$, plagioclase $10 \%$, and biotite $5 \%$.

The $\mathrm{K}$-feldspar is microperthitic, twinned on the Carlsbad law, and partially and variably inverted to microcline (Plate 4, Figure 2). Spectacular vermicular intergrowths with quartz are seen (also elsewhere in the pegmatite). Plagioclase, very near $\mathrm{An}_{20}$ in composition, is twinned on Carlsbad, albite and pericline laws and is rather clouded and sericitized. There is some incipient replacement of plagioclase by $\mathrm{K}$-feldspar near quartz inclusions. Some quartzes have elongated rutile inclusions. The original biotite has been almost completely replaced by very pale green chlorite (with anomalous blue interference colors) causing exsolution of magnetite parallel to the basal plane. 
The upper margin of the pegmatite has a modal composition plagioclase $40 \%$, quartz $35 \%$, K-feldspar $15 \%$, biotite $9 \%$, and zircon $1 \%$. The plagioclase is still near $\mathrm{An}_{20}$ in composition and unzoned. The potassic feldspar shows no twinning and has a very small $2 \mathrm{~V}\left(\sim 10^{\circ}\right.$. $20^{\circ}$ ). This high proportion of plagioclase falls off rapidly away from the margin. At the margin, however, and within the pegmatite at up to $10 \mathrm{~cm}$ from the contact, are large aggregates of purple-red garnet up to $1.5 \mathrm{~cm}$ in diameter. These garnets are intergrown with quartz in vermicular fashion and are surrounded by quartz. They also occur in the granular gneisses for at least 2 meters above the contact and are easily distinguished from the smaller garnets in the gneisses in that they are always much larger and are relatively unaltered. Their paragenesis indicates that they may have grown at the expense of plagioclase, perhaps in the hydrothermal environment associated with the pegmatite intrusion.

A point worth noting vis-a-vis the granite pegmatite is that it shows no sign of deformation. There is no indication of grain reduction. This fact permits separation of veins related to this intrusion from possibly earlier pegmatitic veins emplaced in the gneisses. Several such veins are seen in the gneisses higher in the core sections. Those obviously related to the granite are labeled $\pi_{2}$ in the barrel logs; others, of questionable affinity are labeled $\pi_{1}$. The pegmatitic vein at 16-1, 53-57 $\mathrm{cm}$ is one such vein consisting of $70 \% \mathrm{~K}$-feldspar, $20 \%$ quartz, $10 \%$ plagioclase, and $2 \%$ altered biotite. It shows no grain-size reduction indicative of deformation.

\section{Thin Basic Veins}

Thin intrusive veins cross-cut the foliation of the gneisses in three pieces of Core 16-1, 40-70 cm (Plate 2, Figures 3,4$)$. The upper vein terminates in Piece 4 at 42 $\mathrm{cm}$. Thin quartz-pegmatite layers are also cut by the veins, which appear to have been intruded fairly late in the sequence of events. The veins are dark green in color and are probably of basic composition. The upper vein has been altered to a fine dark green smectite clay (which dried out and cracked apart within a few hours of opening the core), while the lower ones appear to be composed of rather more robust fine-grained chlorite. There is no indication that the veins are deformed.

\section{Microsyenite Intrusion}

The lowermost sample retrieved in the bit consisted of a dark gray fine-grained igneous rock containing xenoliths of granite and quartz. The sample immediately above it carried the chilled margin of this intrusion against the coarse-grained granite. The sample superficially resembles a quartz porphyry, but most of the quartzes are irregular in shape and rather corroded, and are almost certainly xenoliths of gneiss fragments. The groundmass is of almost indeterminate mineralogy, consisting mainly of ? granular plagioclase in a matrix of radiating fibers of ? sericite. The mafic minerals in the groundmass are badly clouded, but some have typical pyroxene forms. There are in addition some chloritized relics of biotite (?) and perfect small hematite hexagons. There are numerous quartzcalcite veins (Plate 4, Figure 6).
The chilled margin showed small laths of plagioclase (5\%), muscovite $(5 \%)$, and $\mathrm{K}$-feldspar? (1\%) in a cryptocrystalline groundmass. The chilled margin is welded rather firmly to the granite in an irregular contact. It differs in this way from the soft altered thin basic veins noted above. The two phases of intrusion are probably unrelated.

\section{GEOCHEMISTRY OF THE BASEMENT ROCKS}

A total of 25 samples of the gneisses, pegmatite, and the microsyenite have been analyzed for major and minor elements in order to provide a greater insight into the nature and possible origin of the rocks. The samples were selected onboard Glomar Challenger after macroscopic and microscopic examination, and chosen to give a wide range of compositional variation.

\section{Sample Preparation and Analysis Technique}

The sample size used $(\sim 60 \mathrm{~g})$ was necessarily much smaller than normally desirable for the geochemical study of coarse-grained rocks. This was a particular difficulty with the granite pegmatite samples, the analyses for which can really only be taken as an indication of the compositional variation within the body. The samples of the finer grained gneisses are, however, probably reasonably representative of the different lithologies.

Crushing was done onboard ship. Rock samples were extracted from the core with the diamond saw, wrapped in aluminum foil, and broken down to centimeter-sized fragments using a hammer and steel platten. Most of the samples were fairly friable, and there is no evidence of significant base-metal contamination. The fragments were then ground to fine powder (ca $60 \mu \mathrm{m}$ ) in the tungsten carbide barrel of the shatterbox swing mill. The only minor contaminants (ppm level) expected at this stage would be W and Co, neither of which have been analyzed for.

The analyses were made using a Philips PW1450 automatic X-ray fluorescence spectrometer and employing a pressed powder disc technique with "Mowiol" as binder. Three separate X-ray tube programs were used to give maximum response and precision for each element:

Cr-tube: $\mathrm{Si}$ Ti Al Fe Mn Mg Ca Na K P S Cl

W-tube: $\mathrm{Nb} \mathrm{Zr} \mathrm{Cr} \mathrm{Ni} \mathrm{Ce} \mathrm{La}$

Mo-tube: Y Sr Rb Th $\mathrm{Pb} \mathrm{Ba} \mathrm{Cu} \mathrm{Zn} \mathrm{Ga}$

Corrections were made for the spectral interference of $\mathrm{Ce}$ on $\mathrm{Ba}$ and $\mathrm{Rb}$ on $\mathrm{Y}$. The interference of $\mathrm{Sr}$ on $\mathrm{Zr}$ was negligible. Values for $\mathrm{Cr}$ may, however, be enhanced slightly by vanadium interference (line overlap). Calibrations for major elements were based on a wide range of wet-analyzed samples (including gneisses) and international standards. The spiking technique (cf. Leake et al., 1969) was used for trace element calibration, together with international standards.

Ferrous iron determinations were carried out using ceric sulfate as a titrant. $\mathrm{Fe}^{2+} / \mathrm{Fe}^{3+}$ ratios may be slightly higher than reported due to the oxidation which takes place on swing mill grinding (Fitton and Gill, 1970). Analysis for $\mathrm{H}_{2} \mathrm{O}$ and $\mathrm{CO}_{2}$ was carried out by the method of Riley (1958) employing a high-temperature 
furnace and separate absorption tubes for $\mathrm{H}_{2} \mathrm{O}$ and $\mathrm{CO}_{2}$. Additional checks for $\mathrm{CO}_{2}$ on the carbonate-rich samples in Section 330-16-1 were made by the backtitration technique after digestion in cold dilute acid. A further check on the volatile content of the samples was made using a Stanton-Redcroft TG-750 thermobalance under controlled atmosphere conditions and heating rates up to $100^{\circ} \mathrm{C}$ per minute. These runs showed that some of the water loss occurred at temperatures not much above $100^{\circ} \mathrm{C}$, presumably from the finegrained degraded biotites and chlorites, while the main bulk of bonded hydroxyl was not liberated completely until $700^{\circ} \mathrm{C}$,

\section{Results}

Complete major and trace element data on the 25 samples of Hole 330 basement analyzed are presented in Table 2 in down-hole order. These comprise $18 \mathrm{sam}$ ples of gneiss, 1 sample of the early $\left(\pi_{1}\right)$ pegmatite, 5 samples of the later $\left(\pi_{2}\right)$ granite pegmatite, and 1 sample of the later microsyenite. Three or four samples at the top of Core 16, Section 1 are calcite impregnated: the geochemistry of these and associated effects in the other samples will be dealt with separately.

\section{Gneisses}

It is clear from the analyses of the gneisses that they can be divided geochemically into three broad groups corresponding to pelite (1 sample), semipelite (8 samples), and quartz-rich semipelite or psammite (6 samples). Means of these three groups together with analyses of the $\pi_{1}$ pegmatite and a mean of four $\pi_{2}$ pegmatite samples are listed in Table 3. Also shown in Table 3 are other mean analyses of various sedimentary rock types and Precambrian gneisses. These are included for comparison to set the data on the Hole 330 basement rocks in a wider context.

The first issue which needs to be clarified is whether or not the geochemical data support the suggested sedimentary origin for the gneisses. It is often difficult under conditions of high-grade metamorphism to elucidate the original nature of the rocks, even with good field exposures. In many well-known Precambrian gneissic terrains particularly, there is still debate as to whether the rocks were originally sediments or volcanics or plutonic intrusions, or even some sort of primeval crust. With the Site 330 basement, "field" data are absent and the issue rests on petrological and geochemical considerations.

The main petrological argument concerns the quartzrich nature of many of the gneisses. There are few igneous rocks where such high quartz contents are possible because of the constraints of solid-liquid phase equilibria. Likewise the high biotite contents of some of the gneisses would be difficult to attain by purely igneous processes except where metasomatic activity has also been important. The absence of a calcic phase such as augite or hornblende in the more melanocratic gneisses is also difficult to explain by igneous mechanisms.

Strong support for a nonigneous origin for the gneisses is afforded by the geochemical data:
1) Five of the gneisses have silica contents of the order of $80 \%$. Such high silica contents are common only in silicified lavas. The majority of gneisses are high in normative quartz and normative corundum and have a mean $\mathrm{tFe} / \mathrm{Mg}$ ratio of $\sim 6$. No igneous rock series displaying a range of $\mathrm{SiO}_{2}$ concentrations similar to that in the gneisses would have so much free quartz or corundum, or have such high $\mathrm{Fe} / \mathrm{Mg}$ ratios.

2) Many igneous rock series have well-defined positive correlations of $\mathrm{K}_{2} \mathrm{O}, \mathrm{Rb}, \mathrm{Ba}, \mathrm{Fe} / \mathrm{Mg}, \mathrm{K} / \mathrm{Na}$ ratios etc with $\mathrm{SiO}_{2}$ and well-marked negative correlations of $\mathrm{MgO}, \mathrm{CaO}, \mathrm{Cr}, \mathrm{Ni}, \mathrm{Sr}$, etc with $\mathrm{SiO}_{2}$. The Site 330 basement gneisses, however, do not show these characteristics: in many cases the correlations are the exact opposite of normal igneous trends (Figures 1-6) or are only very weakly developed. This produces some rather anomalous correlations between some of the other elements. For instance, $\mathrm{K}$ and $\mathrm{Rb}$ correlate positively with $\mathrm{Mg}, \mathrm{Cr}$, and $\mathrm{Ni}-\mathrm{a}$ feature almost impossible to explain by igneous processes.

3) Tarney (1976) and Tarney and Lazell (in preparation) have shown that a plot of $\mathrm{TiO}_{2}$ versus $\mathrm{SiO}_{2}$ can discriminate fairly effectively between gneisses of igneous and sedimentary origin. Rocks of sedimentary origin (provided they are largely clastic and do not contain appreciable chemically precipitated carbonate) plot consistently above an arbitrary line joining $1.85 \%$ $\mathrm{TiO}_{2}$ at $40 \% \mathrm{SiO}_{2}$ with $0 \% \mathrm{TiO}_{2}$ at $81.5 \% \mathrm{SiO}_{2}$, while many igneous rocks in the intermediate to acid range tend to plot below this line. Plotted data for various sediments and metasediments, Archaean orthogneisses, and some calc-alkaline igneous rocks are shown in Figure 7 together with the Site 330 basement data. The Site 330 gneisses clearly plot above the line in the sedimentary field while the $\pi_{1}$ and $\pi_{2}$ pegmatites plot below the line.

It is interesting to compare the geochemistry of the Site 330 gneisses with that of gneisses from early Precambrian shield areas (Table 3, Analyses 13-17). Included in this compilation are mean analyses of the dominant "gray gneisses" from Scotland and Greenland (see also Tarney, 1976), the granulite facies gneisses of Scotland, and the average gneiss compositions of two other shield areas, Canada and Australia. While comparison of "means" can be a rather dubious exercise because of the tendency to smooth out real variations within a population, there are nevertheless some important and real differences between the Site ' 330 gneisses and the Archaean gneisses. Titanium, manganese potash, rubidium, and yttrium are much higher in the Falkland basement rocks, while calcium, sodium, and strontium are much lower. Strontium in particular is almost an order of magnitude lower than in Archaean gneisses. Some of these differences are accentuated if element ratios such as $\mathrm{Rb} / \mathrm{Sr}, \mathrm{Ba} / \mathrm{Rb}$, or $\mathrm{Ba} / \mathrm{Sr}$ are considered, and yet these ratios tend to lie within fairly restricted limits for individual samples within each gneiss population, Site 330 rocks included (Sheraton et al., 1973).

Comparisons with Archaean granulite facies gneisses (Table 3) show even greater differences. Many granulite facies rocks are notably deficient in $\mathrm{K}, \mathrm{Rb}, \mathrm{Th}$, and $\mathrm{U}$. 
TABLE 2

Geochemical Data on Basement Samples From Hole 330, Arranged in Downhole Order

\begin{tabular}{lccccccccccccc}
\hline & 1 & 2 & 3 & 4 & 5 & 6 & 7 & 8 & 9 & 10 & 11 & 12 \\
\hline $\mathrm{SiO}_{2}$ & 67.1 & 71.2 & 66.3 & 79.6 & 78.9 & 70.9 & 43.3 & 70.1 & 63.4 & 59.8 & 58.0 & 64.6 & 81.3 \\
$\mathrm{TiO}_{2}$ & 0.76 & 0.68 & 0.021 & 0.92 & 1.25 & 0.89 & 2.41 & 0.082 & 1.16 & 1.27 & 1.31 & 1.21 & 0.88 \\
$\mathrm{Al}_{2} \mathrm{O}_{3}$ & 7.3 & 6.0 & 12.3 & 6.6 & 6.4 & 9.1 & 20.4 & 14.3 & 13.7 & 15.3 & 18.7 & 13.7 & 7.0 \\
$\mathrm{Fe}_{2} \mathrm{O}_{3}$ & 1.70 & 2.36 & 0.95 & 2.65 & 3.71 & 4.48 & 6.82 & 2.03 & 5.42 & 5.87 & 4.56 & 4.58 & 2.67 \\
$\mathrm{FeO}$ & 1.54 & 1.36 & 0.03 & 0.68 & 2.37 & 2.26 & 7.84 & 2.42 & 3.74 & 3.53 & 3.62 & 3.46 & 1.38 \\
$\mathrm{MnO}$ & 0.151 & 0.160 & 0.115 & 0.108 & 0.123 & 0.118 & 0.132 & 0.121 & 0.110 & 0.124 & 0.114 & 0.120 & 0.116 \\
$\mathrm{MgO}$ & 0.65 & 0.59 & 0.19 & 0.69 & 0.96 & 1.20 & 2.28 & 0.48 & 1.85 & 1.76 & 1.59 & 1.66 & 0.80 \\
$\mathrm{CaO}$ & 9.55 & 8.62 & 6.72 & 2.32 & 0.98 & 2.16 & 1.06 & 0.52 & 0.83 & 1.34 & 0.322 & 0.80 & 0.62 \\
$\mathrm{Na}_{2} \mathrm{O}$ & 0.83 & 0.60 & 1.37 & 1.02 & 0.85 & 1.29 & 0.38 & 0.96 & 0.86 & 1.41 & 0.78 & 1.56 & 2.02 \\
$\mathrm{~K}_{2} \mathrm{O}$ & 2.35 & 2.24 & 6.68 & 2.72 & 2.15 & 3.32 & 6.91 & 5.92 & 3.74 & 4.08 & 6.58 & 3.73 & 1.23 \\
$\mathrm{P}_{2} \mathrm{O}_{5}$ & 0.004 & 0.011 & 0.056 & 0.336 & 0.013 & 0.032 & 0.005 & 0.300 & 0.006 & 0.377 & 0.109 & 0.067 & 0.062 \\
$\mathrm{H}_{2} \mathrm{O}$ & 1.97 & 1.82 & 0.90 & 1.69 & 2.65 & 3.15 & 7.58 & 2.20 & 4.23 & 4.12 & 3.76 & 3.43 & 1.60 \\
$\mathrm{CO}_{2}$ & 5.94 & 4.70 & 4.50 & 1.00 & 0.40 & 0.90 & 0.70 & 0.20 & 0.60 & 0.70 & 0.10 & 0.27 & 0.10 \\
$\mathrm{Total}$ & 99.8 & 100.3 & 100.1 & 100.3 & 100.7 & 99.8 & 99.8 & 99.6 & 99.6 & 99.7 & 99.5 & 99.2 & 99.8
\end{tabular}

Trace Elements (ppm)

$\begin{array}{lrrrr}\mathrm{S} & 129 & 193 & 395 & 108 \\ \mathrm{Cl} & 570 & 490 & 260 & 260 \\ \mathrm{Cr} & 68 & 66 & 17 & 77 \\ \mathrm{Ni} & 30 & 31 & 8 & 24 \\ \mathrm{Cu} & 115 & 180 & 1400 & \\ \mathrm{Zn} & 54 & 56 & 29 & 39 \\ \mathrm{Rb} & 56 & 57 & 214 & 96 \\ \mathrm{Sr} & 67 & 64 & 125 & 62 \\ \mathrm{Y} & 30 & 31 & 21 & 46 \\ \mathrm{Zr} & 256 & 271 & 11 & 428 \\ \mathrm{Nb} & 7 & 8 & 2 & 1 \\ \mathrm{Ba} & 306 & 421 & 594 & 574 \\ \mathrm{La} & 13 & 14 & 6 & 20 \\ \mathrm{Ce} & 26 & 35 & 12 & 46 \\ \mathrm{~Pb} & 11 & 7 & 36 & \\ \mathrm{Th} & 5 & 5 & 1 & \end{array}$

$\begin{array}{rrrr}108 & 158 & 115 & 31 \\ 260 & 360 & 750 & 690 \\ 77 & 117 & 61 & 223 \\ 24 & 28 & 39 & 87 \\ 5 & 20 & 19 & 12 \\ 39 & 72 & 80 & 163 \\ 96 & 68 & 93 & 214 \\ 62 & 42 & 63 & 44 \\ 46 & 19 & 21 & 30 \\ 428 & 549 & 336 & 323 \\ 11 & 14 & 11 & 26 \\ 574 & 608 & 518 & 698 \\ 20 & 20 & 35 & 28 \\ 46 & 35 & 59 & 56 \\ 8 & 9 & 19 & 13 \\ 9 & 11 & 24 & 14\end{array}$

$\begin{array}{rr}64 & 67 \\ 290 & 470 \\ 13 & 106 \\ 18 & 55 \\ 5 & 36 \\ 32 & 137 \\ 161 & 127 \\ 83 & 47 \\ 29 & 17 \\ 72 & 172 \\ 2 & 18 \\ 641 & 354 \\ 10 & 21 \\ 20 & 38 \\ 49 & 14 \\ 6 & 11\end{array}$

$\begin{array}{rrrr}86 & 79 & 121 & 103 \\ 730 & 350 & 390 & 410 \\ 101 & 113 & 91 & 71 \\ 56 & 49 & 91 & 29 \\ 5 & 5 & 8 & 5 \\ 119 & 141 & 138 & 86 \\ 119 & 189 & 118 & 53 \\ 85 & 73 & 78 & 66 \\ 56 & 27 & 28 & 22 \\ 195 & 179 & 189 & 335 \\ 14 & 11 & 12 & 14 \\ 489 & 688 & 527 & 574 \\ 51 & 33 & 30 & 15 \\ 98 & 57 & 54 & 33 \\ 15 & 32 & 17 & 7 \\ 7 & 9 & 7 & 7\end{array}$

CIPW Norms (recalculated dry)

\begin{tabular}{|c|c|c|c|c|c|c|c|c|c|c|c|c|c|}
\hline Q & 48.5 & 53.7 & 30.3 & 60.7 & 62.7 & 46.4 & 6.4 & 39.6 & 39.8 & 31.2 & 23.8 & 36.7 & 62.8 \\
\hline C & 0.0 & 0.0 & 1.1 & 0.8 & 1.9 & 1.8 & 13.2 & 6.2 & 8.7 & 9.1 & 10.6 & 6.8 & 1.7 \\
\hline z & 0.05 & 0.06 & 0.0 & 0.09 & 0.11 & 0.07 & 0.7 & 0.0 & 0.04 & 0.04 & 0.04 & 0.04 & 0.07 \\
\hline Or & 14.2 & 13.5 & 39.9 & 16.3 & 13.0 & 20.4 & 44.4 & 36.0 & 23.3 & 25.3 & 40.7 & 23.1 & 7.4 \\
\hline $\mathrm{Ab}$ & 6.3 & 4.5 & 11.4 & 8.8 & 6.8 & 10.3 & 2.4 & 8.4 & 6.9 & 12.4 & 6.6 & 13.4 & 17.0 \\
\hline An & 0.9 & 7.5 & 4.7 & 3.2 & 2.4 & 5.1 & 1.0 & 0.8 & 0.4 & 0.0 & 0.4 & 2.0 & 2.2 \\
\hline $\mathrm{HI}$ & 0.09 & 0.08 & 0.03 & 0.0 & 0.06 & 0.12 & 0.12 & 0.0 & 0.08 & 0.02 & 0.03 & 0.05 & 0.05 \\
\hline $\mathrm{Di}$ & 0.15 & 4.8 & 0.0 & 0.0 & 0.0 & 0.0 & 0.0 & 0.0 & 0.0 & 0.0 & 0.0 & 0.0 & 0.0 \\
\hline Hy & 4.16 & 2.2 & 1.6 & 3.9 & 7.2 & 9.3 & 19.5 & 6.3 & 13.4 & 13.2 & 11.3 & 11.5 & 5.2 \\
\hline Ol & 0.0 & 0.0 & 0.0 & 0.0 & 0.0 & 0.0 & 0.0 & 0.0 & 0.0 & 0.0 & 0.0 & 0.0 & 0.0 \\
\hline Mt & 1.3 & 1.4 & 0.4 & 1.3 & 2.4 & 2.6 & 6.1 & 1.7 & 3.6 & 3.7 & 3.3 & 3.2 & 1.6 \\
\hline II & 1.5 & 1.3 & 0.04 & 1.8 & 2.4 & 1.8 & 5.0 & 0.2 & 2.3 & 2.5 & 2.6 & 2.4 & 1.7 \\
\hline Ap & 0.01 & 0.03 & 0.13 & 0.8 & 0.03 & 0.08 & 0.01 & 0.7 & 0.01 & 0194 & 0.27 & 0.17 & 0.15 \\
\hline Pr & 0.02 & 0.04 & 0.07 & 0.02 & 0.03 & 0.02 & 0.0 & 0.0 & 0.0 & 0.0 & 0.0 & 0.02 & 0.02 \\
\hline $\mathrm{Cc}_{\mathrm{c}}$ & 13.8 & 10.9 & 10.3 & 2.3 & 0.9 & 2.1 & 1.7 & 0.4 & 1.4 & 1.7 & 0.2 & 0.6 & 0.2 \\
\hline
\end{tabular}

Element Ratios

\begin{tabular}{lcccccccccccrrr}
$\mathrm{K} / \mathrm{Rb}$ & 348 & 326 & 259 & 235 & 262 & 296 & 268 & 305 & 244 & 284 & 289 & 262 & 193 \\
$\mathrm{~K} / \mathrm{Sr}$ & 291 & 290 & 444 & 364 & 425 & 437 & 1300 & 592 & 661 & 398 & 748 & 397 & 155 \\
$\mathrm{~K} / \mathrm{Ba}$ & 64 & 45 & 93 & 39 & 29 & 53 & 82 & 77 & 88 & 69 & 79 & 59 & 18 \\
$\mathrm{~K} / \mathrm{Pb}$ & 1770 & 2660 & 1540 & 2820 & 1980 & 1450 & 4410 & $100 n$ & 2220 & 2260 & 1710 & 1820 & 1460 \\
$\mathrm{Rb} / \mathrm{Sr}$ & 0.84 & 0.89 & 1.71 & 1.55 & 1.62 & 1.48 & 4.86 & 1.94 & 2.70 & 1.40 & 2.59 & 1.51 & 0.80 \\
$\mathrm{Ba} / \mathrm{Sr}$ & 4.6 & 6.4 & 4.8 & 9.3 & 14.5 & 8.2 & 15.9 & 7.7 & 7.5 & 5.7 & 9.4 & 6.8 & 8.7 \\
$\mathrm{Ba} / \mathrm{Rb}$ & 5.5 & 7.2 & 2.8 & 6.0 & 8.9 & 5.6 & 3.3 & 4.0 & 2.8 & 4.1 & 3.6 & 4.5 & 10.8 \\
$\mathrm{Fe} / \mathrm{Mg}$ & 6.08 & 7.61 & 6.00 & 5.72 & 7.66 & 6.75 & 7.90 & 11.40 & 6.00 & 6.45 & 6.26 & 5.88 & 6.09 \\
$\mathrm{Mg} / \mathrm{Cr}$ & 57 & 54 & 67 & 54 & 50 & 119 & 62 & 223 & 105 & 105 & 85 & 110 & 68 \\
$\mathrm{Mg} / \mathrm{Ni}$ & 130 & 115 & 143 & 173 & 207 & 186 & 158 & 161 & 203 & 189 & 196 & 182 & 166 \\
$\mathrm{Ca} / \mathrm{Sr}$ & 1019 & 963 & 384 & 267 & 167 & 245 & 172 & 45 & 126 & 112 & 32 & 73 & 67 \\
\hline
\end{tabular}

Note: The following are sample details. Numbers in brackets are University of Birmingham analysis numbers. 1. [12203] 16-1, 18-22 cm. (\#1) Calcite-impregnated metasedimentary gneiss. Fine-grained calcite. 2. [12204] 16-1, 30-38 cm. (\#3) Calcite-impregnated metasedimentary gneiss with large poikilitic calcites. 3. [12205] 16-1, $52-55 \mathrm{~cm}$. (\#7) Coarse K-feld spar-rich pegmatitic vein $\left(\pi_{2}\right)$, impregnated and veined by calcite. 4. [12206] 16-1, $71-78 \mathrm{~cm}$. (\#10) Quartz-rich granular gneiss. 5. [12207] 16-1, 100-108 cm. (\#13) Quartz-rich metasedimentary gneiss with K-feldspar, biotite, ore, siderite, and garnet. Some calcite veins. 6. [12208] $16-1,142-146 \mathrm{~cm}$. Metasedimentary gneiss with quartz, plagioclase, $\mathrm{K}$-feldspar, biotite, and ore. 7. [12209] 16-2, 1-11 cm. (\#1) Pelitic gneiss, mainly biotite and garnet variably replaced by chlorite. 8. [12210] 16-2, 43-50 cm. (\#5) Deformed early $\left(\pi_{1}\right)$ K-feldspar pegmatite vein, with garnet, quartz, plagioclase, and biotite. 9. [12211] 16-2, 74-78 cm. (\#10) Banded semipelitic gneiss with quartz, biotite, K-feldspar, and plagioclase. 10. [12212] 16-2, 104-108 cm. Banded semipelitic gneiss with quartz, biotite, plagioclase, and garnet. 11 [12213] 16-2,117-124 cm. (\#18) Metasedimentary gneiss with quartz, K-feldspar, biotite, garnet, and tourmaline. 12. [12214] 16-2, 136-144 cm. 
TABLE 2 - Continued

\begin{tabular}{cccccccccccc}
\hline 14 & 15 & 16 & 17 & 18 & 19 & 20 & 21 & 22 & 23 & 24 & 25 \\
\hline 70.5 & 80.9 & 79.7 & 66.9 & 61.7 & 61.0 & 61.8 & 71.6 & 71.5 & 63.0 & 73.9 & 66.3 \\
1.19 & 0.82 & 0.78 & 1.23 & 1.13 & 1.15 & 1.19 & 0.138 & 0.192 & 0.014 & 0.35 & 0.87 \\
13.0 & 7.0 & 7.1 & 13.6 & 15.4 & 15.2 & 15.3 & 15.4 & 15.6 & 21.6 & 13.3 & 13.3 \\
3.36 & 2.53 & 2.77 & 2.73 & 4.23 & 4.60 & 4.39 & 0.66 & 0.86 & 0.10 & 1.37 & 2.04 \\
2.15 & 1.76 & 2.00 & 4.10 & 3.75 & 3.60 & 3.58 & 0.02 & 0.10 & 0.05 & 0.22 & 4.01 \\
1.40 & 0.97 & 0.92 & 2.16 & 1.87 & 0.28 & 1.87 & 0.28 & 0.39 & 0.04 & 0.54 & 2.18 \\
0.24 & 0.73 & 1.21 & 0.56 & 0.78 & 1.65 & 0.47 & 0.67 & 0.44 & 0.73 & 0.32 & 1.01 \\
0.74 & 1.75 & 1.83 & 1.09 & 1.13 & 1.04 & 1.08 & 2.27 & 2.70 & 2.14 & 2.29 & 1.23 \\
4.62 & 1.50 & 1.32 & 4.12 & 4.59 & 4.29 & 5.29 & 7.77 & 6.72 & 10.94 & 6.42 & 4.65 \\
0.053 & 0.062 & 0.072 & 0.085 & 0.108 & 0.029 & 0.111 & 0.133 & 0.125 & 0.148 & 0.122 & 0.089 \\
2.48 & 1.37 & 1.67 & 2.81 & 3.46 & 4.02 & 3.83 & 0.72 & 0.79 & 0.46 & 0.79 & 2.89 \\
0.10 & 0.20 & 0.40 & 0.20 & 0.20 & 0.20 & 0.20 & 0.10 & 0.10 & 0.10 & 0.10 & 0.80 \\
99.9 & 99.8 & 99.9 & 99.5 & 98.5 & 99.0 & 99.3 & 99.8 & 99.6 & 99.4 & 99.8 & 99.5
\end{tabular}

lements (ppm)

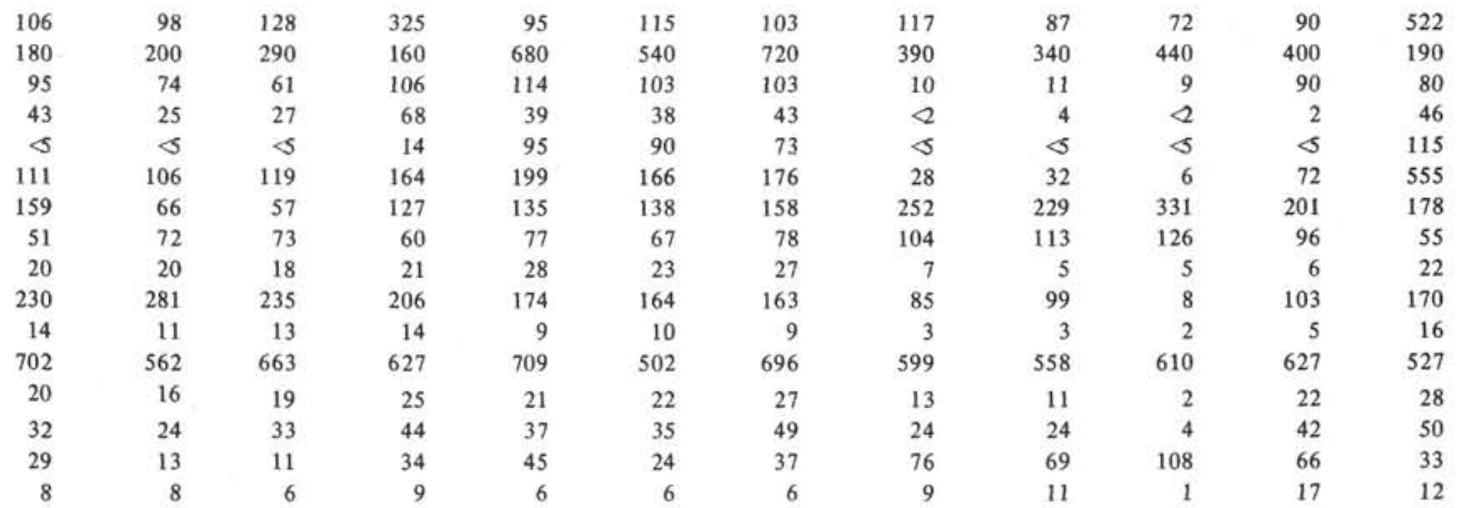

orms (recalculated dry)

$\begin{array}{cccccccccccc}45.7 & 62.2 & 60.7 & 39.7 & 32.8 & 31.4 & 31.1 & 27.5 & 29.2 & 7.7 & 35.0 & 35.1 \\ 6.9 & 1.8 & 1.6 & 7.2 & 8.4 & 6.8 & 8.2 & 2.6 & 3.6 & 5.5 & 2.5 & 4.8 \\ 0.05 & 0.06 & 0.05 & 0.04 & 0.04 & 0.03 & 0.03 & 0.02 & 0.02 & 0.0 & 0.02 & 0.04 \\ 28.1 & 9.0 & 8.0 & 25.2 & 28.6 & 26.8 & 32.9 & 46.4 & 40.3 & 65.4 & 38.4 & 28.7 \\ 6.3 & 14.9 & 15.5 & 9.5 & 9.3 & 8.5 & 8.8 & 19.1 & 22.9 & 18.0 & 19.3 & 10.8 \\ 0.4 & 2.1 & 3.2 & 1.1 & 2.2 & 7.2 & 0.5 & 2.0 & 0.9 & 2.2 & 0.3 & 4.7 \\ 0.02 & 0.02 & 0.03 & 0.0 & 0.09 & 0.09 & 0.09 & 0.03 & 0.02 & 0.03 & 0.03 & 0.01 \\ 0.0 & 0.0 & 0.0 & 0.0 & 0.0 & 0.0 & 0.0 & 0.0 & 0.0 & 0.0 & 0.0 & 0.0 \\ 7.8 & 6.1 & 6.5 & 11.4 & 12.3 & 13.0 & 12.1 & 1.3 & 1.8 & 0.4 & 2.6 \\ 0.0 & 0.0 & 0.0 & 0.0 & 0.0 & 0.0 & 0.0 & 0.0 & 0.0 & 0.0 & 0.0 \\ 2.1 & 1.7 & 1.9 & 2.7 & 3.2 & 3.3 & 3.2 & 0.25 & 0.35 & 0.06 & 0.6 \\ 2.3 & 1.6 & 1.5 & 2.4 & 2.3 & 2.3 & 2.4 & 0.26 & 0.37 & 0.03 & 0.7 \\ 0.13 & 0.15 & 0.17 & 0.21 & 0.27 & 0.07 & 0.28 & 0.32 & 0.30 & 0.35 & 0.29 \\ 0.02 & 0.0 & 0.02 & 0.06 & 0.0 & 0.02 & 0.02 & 0.02 & 0.0 & 0.0 & 0.22 \\ 0.2 & 0.5 & 0.9 & 0.5 & 0.5 & 0.5 & 0.5 & 0.2 & 0.2 & 0.2 & 0.0\end{array}$

\begin{tabular}{|c|c|c|c|c|c|c|c|c|c|c|c|}
\hline 241 & 189 & 192 & 269 & 282 & 258 & 228 & 256 & 243 & 274 & 265 & 217 \\
\hline 752 & 173 & 150 & 570 & 495 & 532 & 563 & 620 & 494 & 721 & 555 & 702 \\
\hline 55 & 22 & 17 & 55 & 54 & 71 & 63 & 108 & 100 & 149 & 85 & 73 \\
\hline 1322 & 958 & 996 & 1005 & 847 & 1480 & 1186 & 849 & 808 & 840 & 807 & 1170 \\
\hline 3.12 & 0.92 & 0.78 & 2.12 & 1.75 & 2.06 & 2.03 & 2.42 & 2.03 & 2.63 & 2.09 & 3.24 \\
\hline 13.8 & 7.8 & 9.1 & 10.5 & 9.2 & 7.5 & 8.9 & 5.8 & 4.9 & 4.8 & 6.5 & 9.6 \\
\hline 4.4 & 8.5 & 11.6 & 4.9 & 5.3 & 3.6 & 4.4 & 2.4 & 2.4 & 1.8 & 3.1 & 3.0 \\
\hline 4.76 & 5.36 & 6.29 & .3 .91 & 5.21 & 4.84 & 5.19 & 2.82 & 2.89 & 4.51 & 3.47 & 3.46 \\
\hline 89 & 79 & 91 & 123 & 99 & 120 & 109 & 169 & 214 & 27 & 325 & 164 \\
\hline 196 & 234 & 206 & 191 & 289 & 327 & 262 & - & 588 & - & 1600 & 286 \\
\hline 34 & 72 & 119 & 67 & 72 & 176 & 43 & 46 & 28 & 41 & 24 & 131 \\
\hline
\end{tabular}

(\#21) Banded semipelitic gneiss with quartz, biotite, plagioclase, and K-feldspar. 13. [12215] 17-1, 46-52 cm. (\#1) Garnet-bearing quartz-rich granular gneiss with plagioclase and biotite. $14 .[12216] 17-1,58-63 \mathrm{~cm}$. ( $\# 3)$ Metasedimentary gneiss with quartz, K-feldspar, biotite, plagioclase, garnet, and tourmaline. 15. [12217] $17-1,78-83 \mathrm{~cm}$. (\#6) Quartz-rich metasedimentary gneiss. 16. [12218] 17-1, 93-102 cm. (\#8) Quartz-rich metasedimentary gneiss. 17. [12219] 17-2, 8-16 cm. (\#3) Foliated metasedimentary gneiss with garnet. 18. [12220] 17-2, 37-45 cm. (\#8) Foliated semipelitic gneiss with garnet. 19. [12221] 17-2, 68-71 cm. (\#14) Foliated semipelitic gneiss. 20. [12222] 17-2, 122-129 cm. (\#23) Foliated semipelitic gneiss. 21. [12223] 17-3, 65-74 cm. (\#11) Granite pegmatite ( $\pi_{2}$ ). 22. [12224] 17-3, $90-100 \mathrm{~cm}$. (\#14) Granite pegmatite $\left(\pi_{2}\right)$. 23. [12225] 17-3, 107-118 cm. (\#16) Granite pegmatite $\left(\pi_{2}\right)$. Coarse-grained K-feldspar-rich facies. 24. [12226] 17, CC (\#1) Granite pegmatite $\left(\pi_{2}\right) .25$. [12227] 17, CC (\#3) Fine-grained microsyenite, with some quartz and granite xenoliths. Sericitised groundmass. Secondary thin veins filled with calcite and quartz. 


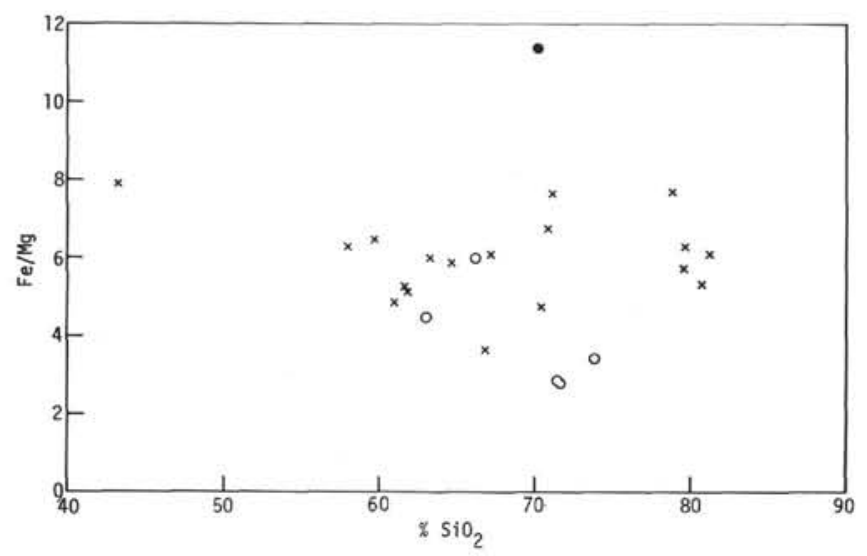

Figure 1. Plot of total $\mathrm{Fe} / \mathrm{Mg}$ versus $\mathrm{SiO}_{2}$ for Site 330 basement rocks. Ornament : crosses, gneisses; filled circle, $\pi_{1}$ pegmatite; open circles, $\pi_{2}$ peginatites.

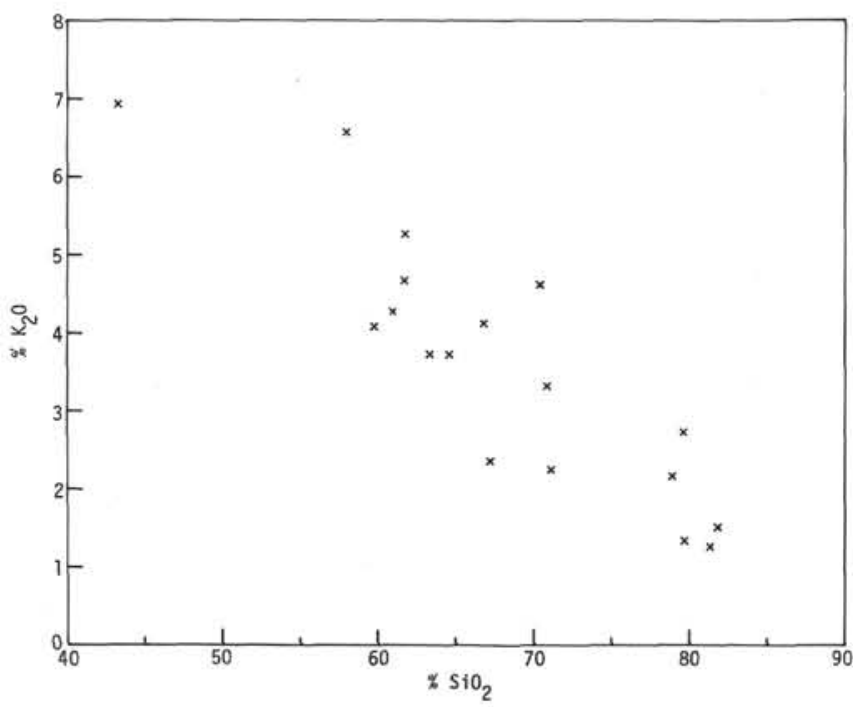

Figure 2. Plot of $\mathrm{K}_{2} \mathrm{O}$ versus $\mathrm{SiO}_{2}$ for Site 330 basement gneisses illustrating negative correlation of $\mathrm{K}_{2} \mathrm{O}$ with $\mathrm{SiO}_{2}$.

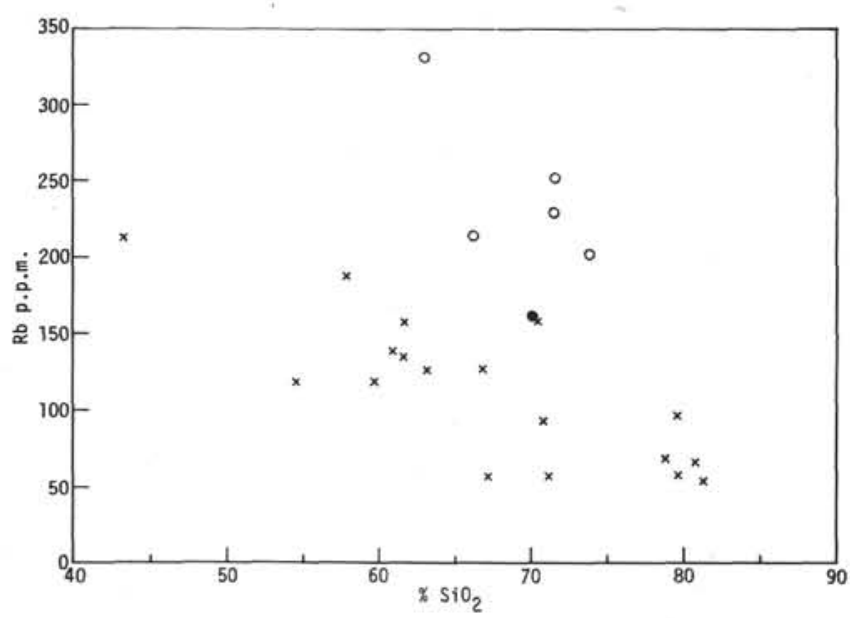

Figure 3. Plot of $\mathrm{Rb}$ versus $\mathrm{SiO}_{2}$ for Site 330 basement rocks. Symbols as in Figure 1. Negative correlation of $\mathrm{Rb}$ with $\mathrm{SiO}_{2}$ in the gneisses. $\pi_{1}$ pegmatite haslower $\mathrm{Rb}$ than $\pi_{2}$ pegmatite samples.

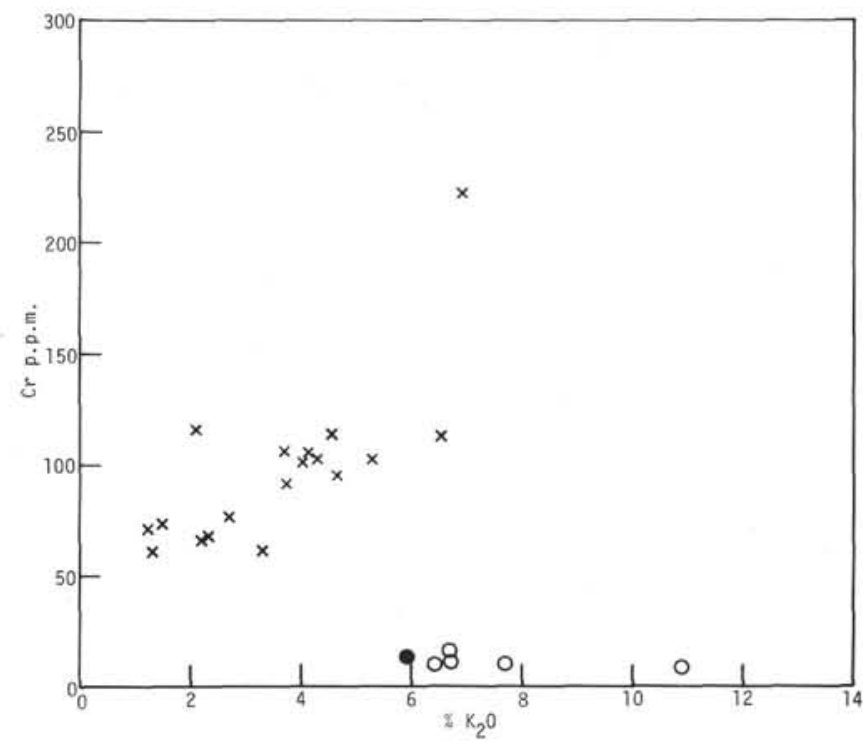

Figure 4. Plot of $\mathrm{Cr}$ versus $\mathrm{K}_{2} \mathrm{O}$ for Site 330 basement rocks, illustrating positive correlation of $\mathrm{Cr}$ with $\mathrm{K}_{2} \mathrm{O}$ in gneisses. Symbols as in Figure 1.

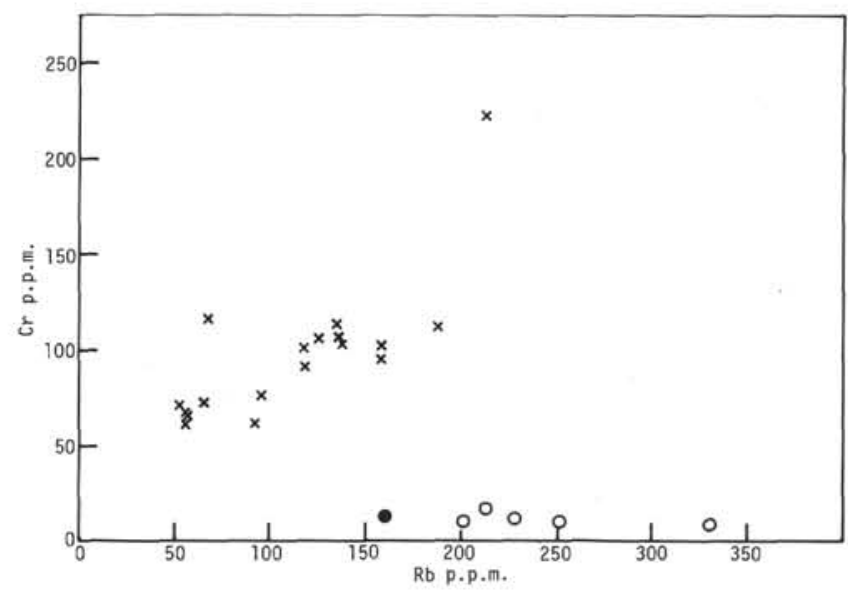

Figure 5. Plot of Cr versus $R b$ for Site 330 basement rocks, illustrating positive correlation of $\mathrm{Rb}$ with $\mathrm{Cr}$ in the gneisses. Symbols as in Figure 1.

compared with their amphibolite facies counterparts (Tarney et al., 1972; Sheraton et al., 1973; Heier, 1973; Tarney, 1976) and as a result, element ratios such as $\mathrm{K} / \mathrm{Rb}$ and $\mathrm{Ba} / \mathrm{Rb}$ are notably high, and ratios such as $\mathrm{Rb} / \mathrm{Sr}, \mathrm{K} / \mathrm{Sr}$ and $\mathrm{K} / \mathrm{Ba}$ characteristically low (Tarney et al., 1972). It is clear that the Hole 330 gneisses, in spite of having suffered high-grade metamorphism which may have approached granulite facies, are very different in composition from Archaean granulites, and have element levels and ratios in some cases a whole order of magnitude or more different. Archaean granulites also tend to have very low ${ }^{87} \mathrm{Sr} /{ }^{86} \mathrm{Sr}$ ratios (Spooner and Fairbairn, 1970; Holland and Lambert, 1975) whereas the Falkland basement gneisses are rich in radiogenic strontium (Beckinsale et al., this volume).

It is clear therefore that although the Site 330 gneisses could well be Precambrian in age (Beckinsale 
TABLE 3

Geochemical Comparison of Hole 330 Basement Average Pelitic Gneiss, Semipelitic Gneiss, Quartz-Rich Semipelitic Gneiss, $\pi_{1}$ Pegmatite, and $\pi_{2}$ Pegmatite with Means of Various Late Precambrian-Palaeozoic Sediments from the British Isles, Phanerozoic and Archaean Graywackes, Mean Amphibolite- and Granulite-Facies Gneisses from the Archaean North Atlantic Craton, and Mean Shield Compositions from Canada and Australia

\begin{tabular}{|c|c|c|c|c|c|c|c|c|c|c|c|c|c|c|c|c|c|}
\hline$(\%)$ & 1 & 2 & 3 & 4 & 5 & 6 & 7 & 8 & 9 & 10 & 11 & 12 & 13 & 14 & 15 & 16 & 17 \\
\hline $\mathrm{SiO}_{2}$ & 43.3 & 62.2 & 78.6 & 70.1 & 72.3 & 53.4 & 53.4 & 72.0 & 79.9 & 76.8 & 66.8 & 64.4 & 66.7 & 67.2 & 61.2 & 64.9 & 67.0 \\
\hline $\mathrm{TiO}_{2}$ & 2.41 & 1.20 & 0.92 & 0.08 & 0.23 & 1.30 & 1.07 & 0.63 & 0.30 & 0.13 & 0.63 & 0.62 & 0.34 & 0.31 & 0.54 & 0.52 & 0.5 \\
\hline $\mathrm{Al}_{2} \mathrm{O}_{3}$ & 20.4 & 15.1 & 7.2 & 14.3 & 14.8 & 24.1 & 22.3 & 13.8 & 10.1 & 10.5 & 13.5 & 15.5 & 16.0 & 15.8 & 15.6 & 14.6 & 14.5 \\
\hline $\mathrm{tFe}_{2} \mathrm{O}_{3}$ & 15.5 & 8.7 & 5.1 & 4.7 & 1.1 & 9.7 & 8.4 & 4.3 & 2.5 & 2.3 & 5.53 & 6.54 & 3.57 & 3.25 & 5.93 & 4.42 & 4.8 \\
\hline $\mathrm{MnO}$ & 0.13 & 0.12 & 0.13 & 0.12 & 0.09 & 0.19 & 0.04 & 0.05 & 0.03 & 0.13 & 0.12 & - & 0.04 & 0.04 & 0.08 & 0.07 & 0.2 \\
\hline $\mathrm{MgO}$ & 2.28 & 1.85 & 0.92 & 0.48 & 0.40 & 1.85 & 2.11 & 1.45 & 1.46 & 0.47 & 2.15 & 3.12 & 1.44 & 1.09 & 3.36 & 2.24 & 2.5 \\
\hline $\mathrm{CaO}$ & 1.06 & 0.84 & 1.34 & 0.52 & 0.48 & 0.89 & 0.92 & 1.28 & 0.15 & 1.40 & 2.54 & 2.22 & 3.18 & 3.54 & 5.57 & 4.12 & 4.0 \\
\hline $\mathrm{Na}_{2} \mathrm{O}$ & 0.38 & 1.12 & 1.46 & 0.96 & 2.42 & 1.15 & 2.02 & 2.63 & 2.20 & 2.20 & 2.93 & 3.74 & 4.90 & 4.74 & 4.42 & 3.46 & 2.5 \\
\hline $\mathrm{K}_{2} \mathrm{O}$ & 6.91 & 4.55 & 2.04 & 5.92 & 6.97 & 3.56 & 3.99 & 3.56 & 3.31 & 4.43 & 1.99 & 2.44 & 2.09 & 2.10 & 1.03 & 3.10 & 3.0 \\
\hline $\mathrm{P}_{2} \mathrm{O}_{5}$ & 0.005 & 0.111 & 0.096 & 0.300 & 0.127 & 0.11 & 0.08 & 0.26 & 0.01 & 0.12 & 0.16 & - & 0.14 & 0.12 & 0.18 & 0.15 & 0.2 \\
\hline \multicolumn{18}{|c|}{ Trace Elements (ppm) } \\
\hline $\mathrm{s}$ & 31 & 124 & 84 & 64 & 98 & 277 & 405 & & & & & & 140 & 230 & 870 & 600 & - \\
\hline $\mathrm{Cl}$ & 690 & 505 & 378 & 290 & 377 & & & & & & & & 170 & 100 & 150 & 100 & \\
\hline $\mathrm{Cr}$ & 223 & 105 & 77 & 13 & 10 & 130 & 194 & 42 & 32 & & & & 32 & 23 & 88 & 99 & \\
\hline $\mathrm{Ni}$ & 87 & 50 & 29 & 18 & $<2$ & 48 & 47 & 17 & 11 & & & 91 & 20 & 13 & 58 & 23 & \\
\hline $\mathrm{Cu}$ & 12 & $\sim 40$ & $\sim 9$ & 5 & $<5$ & 104 & & & & & & & & & & & \\
\hline $\mathrm{Zn}$ & 163 & 155 & 84 & 32 & 88 & & & & & & & & & & & & \\
\hline $\mathrm{Rb}$ & 214 & 124 & 72 & 161 & 227 & 211 & 167 & 112 & 86 & & & 88 & 74 & 44 & 11 & 118 & 115 \\
\hline $\mathrm{St}$ & 44 & 71 & $6 \hat{3}$ & 83 & 104 & 136 & 77 & 260 & 78 & & & 424 & 580 & 580 & 569 & 340 & 135 \\
\hline $\mathbf{Y}$ & 30 & 28 & 24 & 29 & 6 & 25 & 33 & 23 & 8 & & & & 7 & 8 & 9 & & \\
\hline $\mathrm{Zr}$ & 323 & 180 & 361 & 72 & 96 & 219 & 254 & 372 & 169 & & & 196 & 193 & 207 & 202 & 400 & 220 \\
\hline $\mathrm{Nb}$ & 26 & 12 & 12 & 2 & 4 & & & & & & & & 6 & 6 & 5 & & \\
\hline $\mathrm{Ba}$ & 698 & 574 & 583 & 641 & 594 & 890 & 775 & 858 & 690 & & & & 713 & 1040 & 757 & 1070 & 610 \\
\hline $\mathrm{La}$ & 28 & 29 & 21 & 10 & 15 & 45 & 34 & 29 & 10 & & & & 32 & & 20 & & 35 \\
\hline $\mathrm{Ce}$ & 56 & 51 & 38 & 20 & 30 & 98 & 69 & 66 & 26 & & & & 71 & 68 & 48 & & \\
\hline $\mathrm{Pb}$ & 13 & 27 & 11 & 49 & 70 & 61 & 18 & 19 & 11 & & & & 22 & 16 & 13 & & 20 \\
\hline Th & 14 & 8 & 11 & 6 & 12 & 17 & 13 & 7 & 3 & & & & 11 & 10 & 1 & & 3 \\
\hline \multicolumn{18}{|c|}{ Element Ratios } \\
\hline $\mathrm{K} / \mathrm{Rb}$ & 268 & 305 & 235 & 308 & 255 & 140 & 198 & 264 & 319 & - & - & 230 & 234 & 395 & 763 & 220 & 217 \\
\hline $\mathrm{K} / \mathrm{Sr}$ & 1300 & 530 & 270 & 592 & 556 & 217 & 430 & 114 & 350 & - & - & 48 & 30 & 30 & 16 & 75 & 184 \\
\hline $\mathrm{K} / \mathrm{Ba}$ & 82 & 66 & 29 & 77 & 97 & 33 & 43 & 34 & 40 & - & - & - & 24 & 17 & 11 & 24 & 41 \\
\hline $\mathrm{K} / \mathrm{Pb}$ & 4410 & 1400 & 1540 & 1000 & 830 & 480 & 1840 & 1550 & 2500 & - & - & - & 790 & 1100 & 660 & & 710 \\
\hline $\mathrm{Rb} / \mathrm{S} t$ & 4.86 & 1.75 & 1.14 & 1.94 & 2.18 & 1.55 & 2.17 & 0.43 & 1.10 & - & - & 0.21 & 0.13 & 0.08 & 0.02 & 0.35 & 0.85 \\
\hline $\mathrm{Ba} / \mathrm{Sr}$ & 16 & 8.1 & 9.3 & 7.7 & 5.7 & 6.5 & 10.1 & 3.3 & 8.8 & - & - & - & 1.3 & 1.8 & 1.3 & 3.1 & 4.5 \\
\hline $\mathrm{Ba} / \mathrm{Rb}$ & 3.3 & 4.6 & 8.1 & 4.0 & 2.6 & 4.2 & 4.6 & 7.7 & 8.0 & - & - & - & 9.6 & 24 & 68 & 9 & 5.3 \\
\hline $\mathrm{tFe} / \mathrm{Mg}$ & 7.90 & 5.45 & 6.43 & 11.4 & 3.19 & 6.1 & 4.62 & 3.44 & 1.99 & 5.67 & 2.98 & 2.43 & 2.87 & 3.46 & 2.05 & 2.30 & 2.23 \\
\hline $\mathrm{Mg} / \mathrm{Cr}$ & 62 & 106 & 72 & 223 & 240 & 86 & 66 & 208 & 275 & - & - & - & 270 & 280 & 231 & 136 & - \\
\hline $\mathrm{Mg} / \mathrm{Ni}$ & 158 & 223 & 191 & 161 & - & 232 & 271 & 514 & 800 & - & - & 207 & 430 & 510 & 350 & 590 & - \\
\hline $\mathrm{Ca} / \mathrm{Sr}$ & 172 & 85 & 152 & 45 & 33 & 47 & 85 & 35 & 14 & - & - & 37 & 39 & 43 & 70 & 86 & 210 \\
\hline
\end{tabular}

Note: 1. Pelite, Hole 330 basement (Table 2, No. 7). 2. Semipelite, Hole 330 basement (Table 2, mean of No. 9, 10, 11, 12, 17, 18, 19, 20). 3. Quartz-rich semipelite, Hole 330 basement (Table 2, mean of No. 4, 5, 6, 13, 15, 16), 4. Early ( $\left.\pi_{1}\right)$ pegmatite, Hole 330 basement (Table 2, No. 8 ). 5 . Granite pegmatite $\left(\pi_{2}\right)$, Hole 330 basement (Table 2, mean of No. 3, 21, 22, 24). 6. Pelite (mean of 33 samples), Arenig Skiddaw Slate, England (Jeans, 1973). 7. Pelite (mean of 30 samples), Tremadocian Slate, North Wales (Parry, 1970). 8. Quartzitic semipelite (mean of 28 samples) Loch na Dal Beds, Torridonian, Skye (Stoakes, 1971). 9. Arkosic sandstone (mean of 30 samples) Applecross Formation, Torridonian, Scotland (Stoakes, 1971). 10. Average arkose (Pettijohn, 1963). 11. Average graywacke (Pettijohn, 1953). 12. Archaean graywacke (mean of 25 samples) Wyoming (Condie, 1967). 13. Archaean gray gneiss (mean of 39 samples) Lewisian, Scotland (Tarney et al., 1972). 14. Archaean gray gneiss (mean of 41 samples) East Greenland (Tarney et al., 1972). 15. Archaean granulite facies gneiss (mean of 254 acid to basic gneisses) Assynt, Scotland (Sheraton, 1970). 16. Average composition of Canadian Precambrian Shield (Shaw et al., 1967). 17. Average compositi n of Archaean S. W. Australian Shield (Lambert and Heier, 1968).

et al., this volume) there is little geochemical affinity with the majority of Archaean gneisses. This may imply nothing more than the fact that their origin might be quite different. Recent evidence (Moorbath, 1975; Tarney, 1976) suggests that large areas of gneiss terrain in the Archaean may be plutonic and mantle derived. The geochemical evidence presented above suggests, on the other hand, that the Falkland Plateau gneisses are metamorphosed sediments.

The next point for consideration concerns the nature of the sediments. Allowance must be made of course for possible geochemical changes associated with their metamorphism and the later hydrothermal activity, but this may not have affected all elements. Compared with the average graywacke (Pettijohn, 1963) or Archaean Wyoming graywacke (Condie, 1967), the gneisses are significantly poorer in sodium, calcium, and strontium, but richer in potash and rubidium, and have a higher $\mathrm{Fe} / \mathrm{Mg}$ ratio (cf. Table 3; Analyses 11, 12). On the other hand, comparisons with more mature sediments such as shales (Table 3; Analyses 9 and 10) are much closer both for major elements and trace elements, and element ratios. There is no element or element ratio which needs to be changed significantly to allow derivation of the Falkland Plateau gneisses from a mixed shale-siltarkose sedimentary succession. Perhaps the potash content of the biotite pelite $(330-16-1,1)$ is higher than in a typical shale, but this could have been enhanced during 


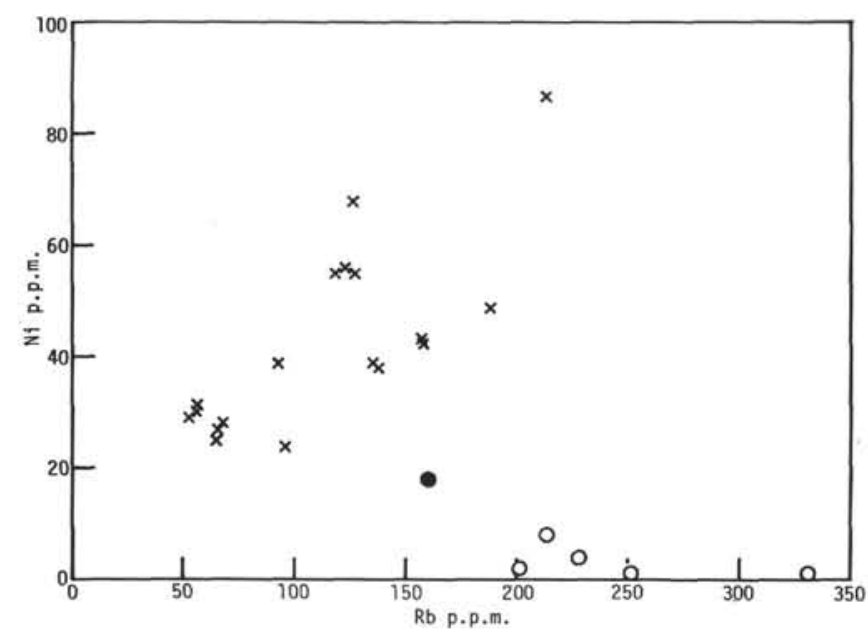

Figure 6. Plot of Ni versus Rb for Site 330 basement rocks. Symbols as in Figure 1. Strong positive correlation of $R b$ with $\mathrm{Ni}$ in the gneisses. $\pi_{1}$ pegmatite has higherNi content than $\pi_{2}$ pegmatite samples.

the metamorphic episode synchronous with growth of the biotite.

The rather low abundance of calcium, sodium, and strontium in the gneisses is explicable either in terms of the maturity of the original sediments, or in terms of the nature of the source terrain, or both. Plagioclase, pyroxenes, and amphiboles, which carry most of the $\mathrm{Ca}, \mathrm{Na}$, and $\mathrm{Sr}$ in igneous rocks, are degraded rapidly during the weathering cycle, and the $\mathrm{Ca}, \mathrm{Na}$, and $\mathrm{Sr}$ ions released are removed easily in solution, while $\mathrm{K}$, $\mathrm{Rb}, \mathrm{Ba}$, etc remain in the less-weathered $\mathrm{K}$-feldspars and micas and equivalent clay minerals. The rather high $\mathrm{K} / \mathrm{Sr}, \mathrm{Rb} / \mathrm{Sr}$, and $\mathrm{Ba} / \mathrm{Sr}$ ratios of the gneisses would conform with mature sediments of this type. In particular the divalent ions $\mathrm{Ba}$ and $\mathrm{Sr}$ are strongly differentiated during the weathering/sedimentary cycle. This is illustrated in Figure 8 with a range of continental sediments from the British Isles. Graywackes have moderately high contents of $\mathrm{Ba}$ and $\mathrm{Sr}$, but with increasing maturity $\mathrm{Sr}$ is partitioned into calcareous sediments and $\mathrm{Ba}$ into pelites and psammites. The Site 330 gneisses clearly correspond with mature pelites and psammites. On the other hand, a source terrain rich in potassic granite would also provide arkosic sediments rich in $\mathrm{K}, \mathrm{Rb}$, and $\mathrm{Ba}$ without the need for extensive weathering.

\section{Pegmatites $\left(\pi 1\right.$ and $\left.\pi_{2}\right)$}

The geochemical differences between the earlier deformed $\pi_{1}$ pegmatite veins and the later $\pi_{2}$ granite pegmatite can be seen by comparing Analyses 4 and 5 in Table 3. Both pegmatites are rich in normative orthoclase and quartz, and have a high normative $\mathrm{Ab} / \mathrm{An}$ ratio. As a result, element values dependent on these minerals are not appreciably different. However, the $\pi_{1}$ pegmatite is significantly richer in $\mathrm{Fe}_{2} \mathrm{O}_{3}, \mathrm{Ni}$, and Y. These differences can be related directly to the presence of garnet in the $\pi$ । pegmatites, a phase which is almandine-rich and is known to favor yttrium and the heavy rare-earth elements in its structure. It is likely of course that these higher values are not original, but may have been enhanced during open-system conditions attending metamorphic growth of the garnets. The $\mathrm{K} / \mathrm{Rb}$ ratio of the $\pi_{1}$ pegmatite is notably higher than that of the $\pi_{2}$ pegmatite, a feature which may be connected with the high metamorphic grade of the former (Tarney et al., 1972).

The five analyses of the later granite pegmatite (Analyses 3, 21, 22, 23, and 24 in Table 2) are rather variable, and this is reflected also in the normative proportions of the major minerals. This partly results from the coarse grain size of the pegmatite and the rather small size of the samples taken from the core for analysis, but the pegmatite itself is rather variable in texture and modal proportions. The $\mathrm{Pb}$ content of the pegmatite is much higher than that in the gneisses, and arises from the substitution of $\mathrm{Pb}$ for $\mathrm{K}$ in the orthoclase structure. As a result $\mathrm{K} / \mathrm{Pb}$ ratios are much lower than in the gneisses.

\section{Microsyenite}

Initially, owing to its rather dark color, this rock was labeled as a microdiorite. Little of its original nature can be deciphered from thin section. Chemically it is fairly rich in $\mathrm{K}_{2} \mathrm{O}$, has a high $\mathrm{K} / \mathrm{Na}$ ratio, is fairly low in $\mathrm{CaO}$ and $\mathrm{Sr}$, and has a high $\mathrm{Rb} / \mathrm{Sr}$ ratio. Normatively it is rich in $\mathrm{K}$-feldspar and quartz and would probably correspond more closely to a quartz-rich syenite than a diorite. The possibility that it may have lamprophyric affinities must be borne in mind, although this would be difficult to reconcile with its geochemistry. However, the intrusion carries small quartz and granite xenoliths, is obviously altered judging from the high water and $\mathrm{CO}_{2}$ contents, has secondary vugs and veins filled with quartz and calcite, and has the highest $\mathrm{Zn}$ and $\mathrm{S}$ contents of any rock in the core. Its composition may therefore have been affected by late hydrothermal activity, and its true parentage must remain in doubt.

\section{MINERAL COMPOSITIONS}

Microprobe determinations of mineral compositions in several of the Hole 330 gneiss samples were made using the Cambridge Microscan V Microanalyzer at Leicester University. The measurements were undertaken by Mr. R.N. Wilson. Correction procedures were carried out using a version of MAGIC IV by J.W. Colby.

The main objective was a determination of the compositions of the main mineral phases, biotite, tourmaline, garnet, plagioclase, and $\mathrm{K}$-feldspar, and in particular an assessment of the compositional differences between red and green biotites. In each case the values quoted are means of between 4 and 10 spot determinations on each crystal or crystal aggregate.

\section{Biotites}

The range in color of the biotites in the gneisses is wide. The primary biotites have a rather intense reddish-brown color, and these are seen to be replaced 

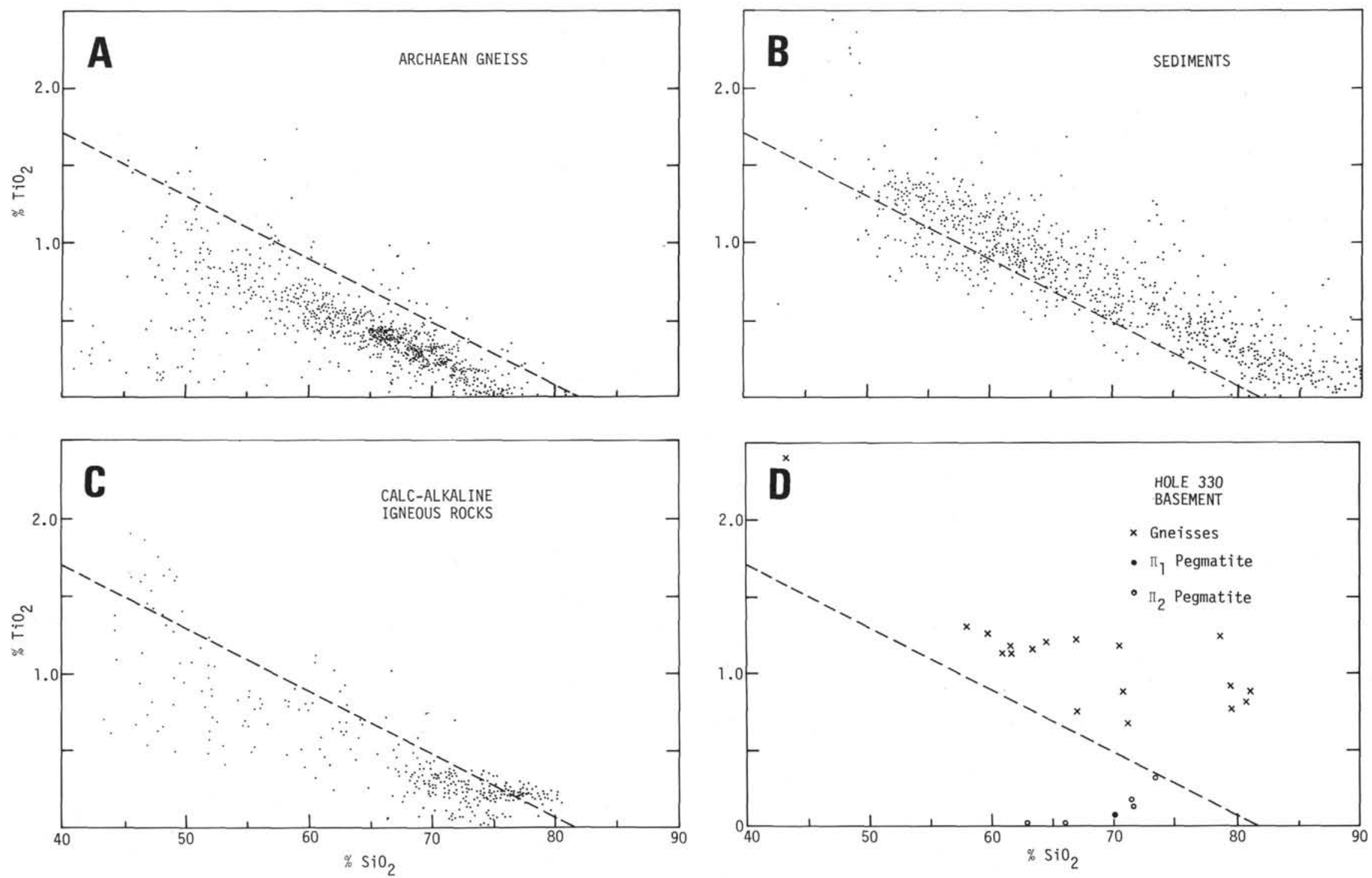

Figure 7. $\mathrm{TiO}_{2} \mathrm{SiO}_{2}$ discrimination plot for separating gneisses of metasedimentary and metaigneous parentage. (a.) Orthogneisses from the North Atlantic Archaean craton. (b.) Clastic sediments of various ages from the British Isles. (c.) Calcalkaline igneous rocks from varying localities in the British Isles. (d.) Site 330 rocks. 

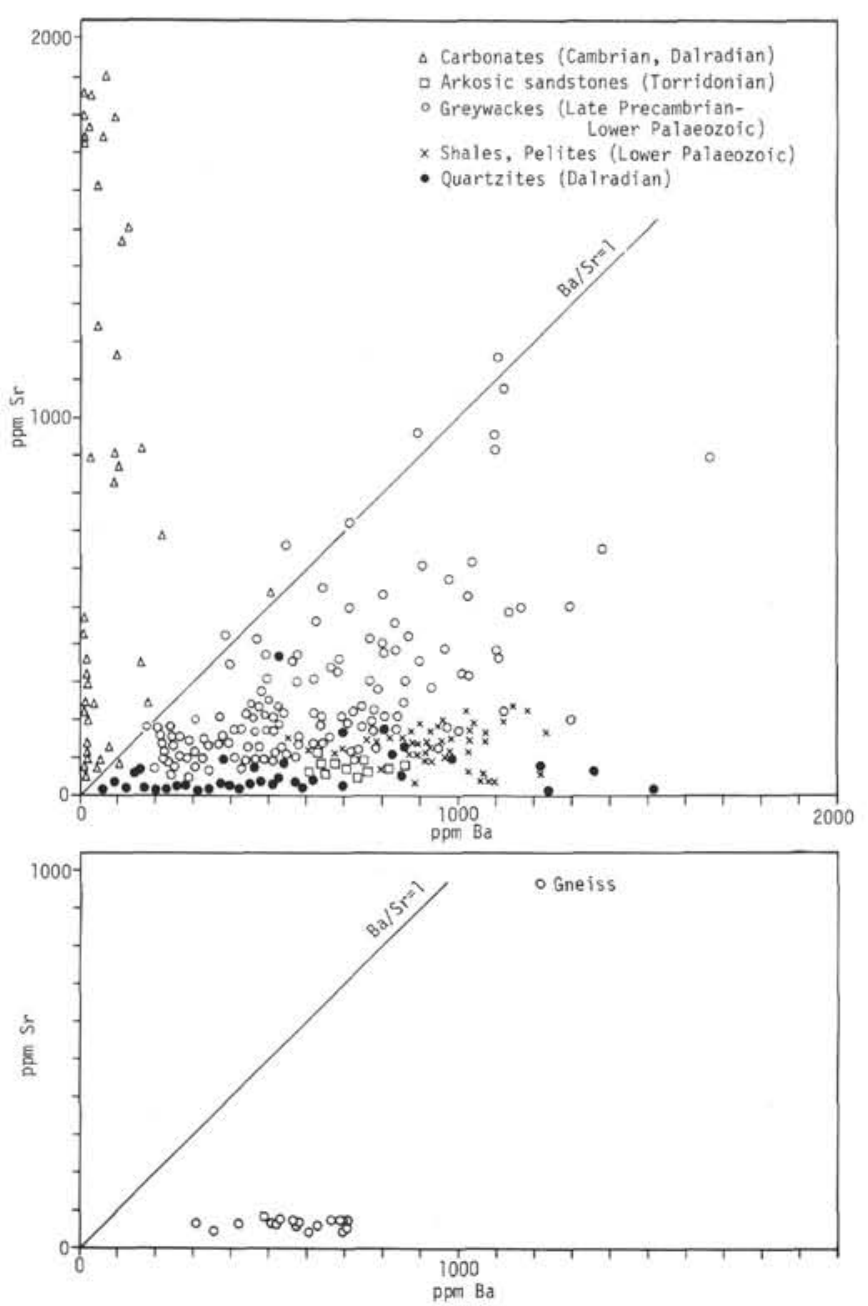

Figure 8. Ba-Sr distributions. (a) Sediments from the British Isles ranging from immature graywackes to mature shales, quartzites, arkose, and limestone. (b) Site 330 basement gneisses. The latter correspond with mature sediments. by a series of green biotites which range in color from quite deep green to almost colorless. Some brown biotites seem to become rather paler brown in color before being replaced by green biotite. The replacement may affect the whole crystal, or only part of it, or individual layers may be replaced, resulting in interbanded redbrown and green varieties. The proportion of reddishbrown biotite remaining in the gneisses is small, but variable: some sections showed no remnant reddishbrown variety.

Microprobe analyses of a series of red-brown, pale brown, green, and very pale green varieties of biotites from three different rocks are shown in Table 4 . The summations of the analyses are rather low, even allowing for the maximum possible $\mathrm{H}_{2} \mathrm{O}, \mathrm{Cl}$, and $\mathrm{F}$ contents, but this is in part due to the difficulty of providing a sufficiently high polish on biotites in the sections peppered with small hard garnet grains. The lower totals of the green and pale-colored biotites are probably real, and reflect the increase in water content in transition towards chlorite.

A feature of the primary red-brown biotites is the high tenor of titanium. Such high values are uncommon in regional metamorphic rocks of low to moderate grade, but $\mathrm{TiO}_{2}$ contents in excess of $4 \%$ are found in biotite from high-grade metamorphic gneisses (e.g., Adirondacks; Engel and Engel, 1960) and in some igneous rocks. Titanium content of biotites is known to increase with metamorphic grade (Deer, et al., 1962), and there is often a corresponding increase in color intensity. The titanium content falls by a factor of about 10 , however, in the green biotites, and this is accompanied by growth of titanomagnetite between the biotite laths or as small inclusions within the laths. If $\mathrm{Ti}$ content is directly related to metamorphic grade, the development of green biotite at the expense of redbrown biotite implies a marked lowering of grade.

There is also a marked change in potash content of the biotites accompanying the transformation from brown to green. The $\mathrm{K}_{2} \mathrm{O}$ values in the red-brown

TABLE 4

Microprobe Analyses of Brown Biotites, Green Hydrobiotites and Chlorite

\begin{tabular}{lrrrrrrrrrr}
\hline \multicolumn{1}{c}{1} & \multicolumn{1}{c}{2} & \multicolumn{1}{c}{3} & \multicolumn{1}{c}{4} & \multicolumn{1}{c}{5} & \multicolumn{1}{c}{6} & 7 & 8 & 9 & 10 \\
\hline $\mathrm{SiO}_{2}$ & 35.14 & 35.40 & 35.96 & 37.42 & 37.10 & 40.05 & 38.33 & 39.57 & 40.79 & 27.13 \\
$\mathrm{TiO}_{2}$ & 4.95 & 4.86 & 4.38 & 4.29 & 1.34 & 0.44 & 0.62 & 0.13 & 0.53 & 0.26 \\
$\mathrm{Al}_{2} \mathrm{O}_{3}$ & 17.01 & 16.89 & 16.92 & 17.16 & 17.30 & 20.50 & 19.95 & 20.37 & 24.49 & 20.82 \\
$\mathrm{tFeO}$ & 18.47 & 18.26 & 18.10 & 16.34 & 19.60 & 17.15 & 19.87 & 18.90 & 12.46 & 27.62 \\
$\mathrm{MnO}$ & 0.04 & 0.05 & 0.06 & 0.05 & 0.02 & 0.01 & 0.03 & 0.04 & 0.03 & 0.11 \\
$\mathrm{MgO}$ & 9.48 & 9.52 & 9.38 & 7.98 & 5.46 & 5.86 & 5.84 & 5.93 & 6.61 & 10.62 \\
$\mathrm{CaO}$ & 0.00 & 0.01 & 0.02 & 0.06 & 0.10 & 0.07 & 0.14 & 0.11 & 0.17 & 0.03 \\
$\mathrm{Na}_{2} \mathrm{O}$ & 0.16 & 0.18 & 0.17 & 0.23 & 0.11 & 0.12 & 0.14 & 0.11 & 0.26 & 0.02 \\
$\mathrm{~K}_{2} \mathrm{O}$ & 8.78 & 8.82 & 8.71 & 7.91 & 4.36 & 5.53 & 3.53 & 3.87 & 6.66 & 0.38 \\
$\mathrm{Total}$ & 94.04 & 93.97 & 93.70 & 91.42 & 85.38 & 89.72 & 88.45 & 89.03 & 92.00 & 86.99 \\
\hline
\end{tabular}

Note: 1. Red-brown biotite, Semipelitic gneiss (17-1 \#3B). 2. Red-brown biotite, Semipelitic gneiss (17-1 \#3B). 3. Red-brown biotite, Semipelitic gneiss (16-2 \#18B). 4. Pale brown biotite, Semipelitic gneiss (16-2 \#18B). 5. Green hydrobiotite, Semipelitic gneiss (17-1 \#3B). 6. Green hydrobiotite, Semipelitic gneiss (17-1 \#3B). 7. Green hydrobiotite, Semipelitic gneiss (16-2 $\# 18 B)$. 8. Green hydrobiotite, Semipelitic gneiss (16-2 \#18B). 9. Pale green biotite, Semipelitic gneiss (16-2 \#18B). 10. Chlorite replacing garnet, $\pi_{1}$ pegmatite (16-2 \#5B). 
biotites are close to the normal stoichiometric levels, but are almost half this value in the green biotites. The resultant charge deficiency must either be compensated by substitution of hydroxonium ion $\left(\mathrm{H}_{3} \mathrm{O}^{+}\right)$for potassium, or by interlayering of biotite with chlorite, vermiculite, or smectite. Certainly the lower summations of the green biotites would correlate with a higher water content required for hydroxonium substitution, but chlorites and clay minerals also have higher water contents than biotite. There is a rather higher alumina content in the green biotites which might accord with interlayer chlorite; on the other hand, $\mathrm{SiO}_{2}$ would be expected to be lower in the green biotites if this were the case, but silica is in fact $4 \%$ higher in the green variety, a feature which might accord better with interlayer smectite. Other chemical differences accompanying the color change are the lowering of $\mathrm{MgO}$ and slight rise in $\mathrm{tFeO}$, with consequent increase of $\mathrm{Fe} / \mathrm{Mg}$ ratio. There is, however, no appreciable difference in $\mathrm{CaO}$ or $\mathrm{Na}_{2} \mathrm{O}$ contents which might be expected to correlate with increasing smectite content. It would appear therefore that there is no positive evidence in favor of an increase in interlayer chlorite, vermiculite or smectite, hence the green variety might be loosely regarded as a "hydrobiotite."

Some of the pale green micaceous material (e.g., Analysis 10 in Table 4) has the low- $-\mathrm{SiO}_{2}$ and low- $\mathrm{K}_{2} \mathrm{O}$ characteristics of chlorite. Similar material is seen replacing the garnets in the upper part of the core. The biotites in the $\pi_{2}$ pegmatites seem to be replaced by chlorite rather than hydrobiotite.

Biotites in the gneisses therefore record a phase of high-grade metamorphism followed by a phase of lower temperature hydrothermal retrogression. This latter event is not, however, connected with the ground-water activity associated with the calcrete formation since both red and green biotites are preserved intact in the most heavily calcreted zone.

\section{Garnets}

The compositions of the garnets from four different rocks are shown in Table 5. Included in the compilation are analyses of both types of garnets in the core. Surprisingly there is negligible compositional difference between the garnets. Moreover individual spot determinations across the crystals in each case are more or less identical and essentially within the range of instrumental error. This implies that the crystals are unzoned and have reached complete equilibrium with their immediate environment. Furthermore the fact that there is no compositional difference between garnets in different samples would seem to indicate that there is to a large extent chemical equilibrium within this part of the core. This is readily understandable, of course, if the garnets have developed from, and in equilibrium with, the primary red-brown biotites because these biotites are themselves compositionally uniform.

The composition of the garnets corresponds closely to almandine. Equivalent garnet compositions are found in metamorphic rocks ranging up to granulite facies, since it is primarily the whole-rock composition
TABLE 5

Microprobe Analyses of Garnets

\begin{tabular}{lrrrr}
\hline & \multicolumn{1}{c}{1} & \multicolumn{1}{c}{2} & \multicolumn{1}{c}{3} & \multicolumn{1}{c}{4} \\
\hline $\mathrm{SiO}_{2}$ & 37.5 & 37.90 & 37.65 & 38.09 \\
$\mathrm{TiO}_{2}$ & 0.02 & 0.02 & 0.01 & 0.02 \\
$\mathrm{Al}_{2} \mathrm{O}_{3}$ & 21.52 & 21.50 & 21.64 & 21.78 \\
$\mathrm{FeO}$ & 32.94 & 33.29 & 33.60 & 33.16 \\
$\mathrm{MnO}$ & 1.34 & 1.15 & 0.76 & 1.13 \\
$\mathrm{MgO}$ & 4.63 & 5.14 & 5.15 & 5.50 \\
$\mathrm{CaO}$ & 0.74 & 0.80 & 0.72 & 0.61 \\
Total & 98.54 & 99.80 & 99.35 & 100.28 \\
\hline
\end{tabular}

Note: 1. Granular garnets in $\pi_{1}$ pegmatite (16-2, 5B). 2. Garnet in semi-pelitic gneiss $(16-2,18 \mathrm{~B}) .3$. Garnet in semi-pelitic gneiss (17-1, 3B). 4. Large garnets in semipelitic gneiss near $\pi_{2}$ pegmatite contact $(17-3,5 B)$.

which determines the garnet composition. In this case the host rocks are low in calcium and have a relatively high $\mathrm{Fe} / \mathrm{Mg}$ ratio, hence the grossular and pyrope components of the garnets are small. Compared with the coexisting biotites, the garnets have a higher $\mathrm{Fe} / \mathrm{Mg}$ ratio. Titanium is strongly partitioned into the biotites while manganese and calcium are equally strongly partitioned into the garnets.

\section{Tourmaline}

Tourmaline occurs in several of the thin sections of the cored gneisses and is a prominent constituent in the loose sand overlying the basement. There is no doubt therefore that it is an important, if minor, component of the gneisses. Most of the tourmaline seems to be associated with the pelitic rather than the psammitic gneisses, but this is understandable in that tourmaline is an aluminous phase.

Analyses of tourmalines from Samples 17-1 (\#3B) and 16-2 (\#18B) are given in Table 6. The analysis summations are about $15 \%$ too low, but this is because, for obvious reasons, no determination could be made for

TABLE 6

Microprobe Analyse of Tourmalines

\begin{tabular}{lrr}
\hline & \multicolumn{1}{c}{1} & \multicolumn{1}{c}{2} \\
\hline $\mathrm{SiO}_{2}$ & 35.33 & 35.62 \\
$\mathrm{TiO}_{2}$ & 1.18 & 1.20 \\
$\mathrm{Al}_{2} \mathrm{O}_{3}$ & 32.61 & 33.10 \\
$\mathrm{tFeO}$ & 5.84 & 5.88 \\
$\mathrm{MnO}$ & 0.00 & 0.02 \\
$\mathrm{MgO}$ & 6.45 & 6.35 \\
$\mathrm{CaO}$ & 1.04 & 1.04 \\
$\mathrm{Na}_{2} \mathrm{O}$ & 1.92 & 1.94 \\
$\mathrm{~K}_{2} \mathrm{O}$ & 0.08 & 0.07 \\
\hline $\mathrm{Total}^{\mathrm{a}}$ & 84.45 & 85.21 \\
$\mathrm{a}^{\mathrm{T} o t a l s}$ & do not \\
$\mathrm{B}_{2} \mathrm{O}_{3}, \mathrm{H}_{2} \mathrm{O}$, F. &
\end{tabular}


$\mathrm{B}_{2} \mathrm{O}_{3}$ (normally $\sim 10.5 \%$ ) or $\mathrm{H}_{2} \mathrm{O}$ of $\mathrm{F}$ (normally $\sim 4 \%$ ). The compositions of the two crystals correspond very closely and they are clearly members of the dravite [ $\left.\mathrm{NaMg}_{3} \mathrm{Al}_{6} \mathrm{~B}_{3} \mathrm{Si}_{6} \mathrm{O}_{27}(\mathrm{OH}, \mathrm{F})_{4}\right]$ schorl $\left[\mathrm{NaFe}_{3} \mathrm{Al}_{6} \mathrm{~B}_{3} \mathrm{Si}_{6} \mathrm{O}_{27}(\mathrm{OH}, \mathrm{F})_{4}\right]$ series, and lie more or less midway between the two end members. The $\mathrm{Mg} / \mathrm{Fe}$ ratio of the tourmalines is much higher than that of the coexisting biotites or garnets. The abundance levels of the minor components $\mathrm{TiO}_{2}, \mathrm{MnO}, \mathrm{CaO}$, and $\mathrm{K}_{2} \mathrm{O}$ are quite normal for tourmalines of the dravite-schorl series.

Although tourmaline is a relatively common detrital mineral in sediments, it is unlikely that this is the explanation for its occurrence in the gneisses, firstly on the grounds that there is rather too much of it, and secondly because detrital tourmaline might be expected to be more abundant in the psammitic rather than the pelitic gneisses. Most occurrences of tourmaline in metamorphic rocks are related to boron metasomatism connected with adjacent granites or pegmatites. In the present case the fact that the tourmalines lie within the gneiss foliation and are apparently syntectonic would seem to rule out connection with the $\pi_{2}$ pegmatite or the microsyenite. They may be related to the $\pi$ 1 pegmatite veins or to some other predeformational granitic body but there is no positive evidence to support either suggestion. Nevertheless the tourmalines signify a further event in the geological history of the gneisses of the Falkland Plateau basement.

\section{Plagioclases}

The plagioclase compositions correspond to sodic oligoclase (Table 7). This is not surprising considering the calcium-poor nature of the gneisses and the fact that another Ca-bearing phase (garnet) is also present. Whether the probed compositions are representative of the primary plagioclase compositions is difficult to say: most of the plagioclases are clouded and replaced by calcite and it is only the more sodic plagioclases in the basement rocks which have evaded this secondary alteration.

\section{Potassic Feldspars}

K-feldspar compositions (Table 8) are not especially unusual. The soda contents, indicating an albite com-

TABLE 7

Microprobe Analyses of Plagioclase

\begin{tabular}{lrrr}
\hline & \multicolumn{1}{c}{1} & \multicolumn{1}{c}{2} & \multicolumn{1}{c}{3} \\
\hline $\mathrm{SiO}_{2}$ & 66.23 & 66.77 & 66.25 \\
$\mathrm{Al}_{2} \mathrm{O}_{3}$ & 21.22 & 20.62 & 21.99 \\
$\mathrm{CaO}$ & 2.00 & 1.17 & 1.98 \\
$\mathrm{Na}_{2} \mathrm{O}$ & 10.65 & 10.70 & 9.13 \\
$\mathrm{~K}_{2} \mathrm{O}$ & 0.12 & 0.15 & 0.72 \\
Total & 100.22 & 99.41 & 100.07 \\
\hline
\end{tabular}

Note: 1. Semipelitic gneiss (16-2, 18B). 2. Semipelitic gneiss (17-1, 3B). $3 . \pi_{1}$ pegmatite $(16-2,5 B)$.
TABLE 8

Microprobe Analyses of Potassic Feldspars

\begin{tabular}{lrrrrr}
\hline & 1 & \multicolumn{1}{c}{2} & \multicolumn{1}{c}{3} & \multicolumn{1}{c}{4} & \multicolumn{1}{c}{5} \\
\hline $\mathrm{SiO}_{2}$ & 64.66 & 64.17 & 64.49 & 64.65 & 64.47 \\
$\mathrm{Al}_{2} \mathrm{O}_{3}$ & 18.46 & 18.29 & 18.95 & 18.36 & 18.29 \\
$\mathrm{CaO}$ & 0.10 & 0.18 & 0.19 & 0.12 & 0.04 \\
$\mathrm{Na}_{2} \mathrm{O}$ & 2.00 & 1.76 & 2.38 & 2.65 & 2.32 \\
$\mathrm{~K}_{2} \mathrm{O}$ & 12.88 & 13.14 & 12.04 & 11.93 & 12.42 \\
Total & 98.10 & 97.54 & 98.05 & 97.71 & 97.54 \\
\hline
\end{tabular}

Note: 1, 2. Semipelitic gneiss (16-2, 18B). 3-5. Semipelitic gneiss $(17-1,3 \mathrm{~B})$.

ponent in solid solution, are however higher than in many metamorphic orthoclases or microclines. K-feldspars of igneous origin may of course have higher soda contents, but considering the soda-poor nature of the gneisses, the high soda contents may be of some significance and indicate relatively high temperature crystallization. Optically the $\mathrm{K}$-feldspars are orthoclases not microcline, and the $2 \mathrm{~V}$ values are very low, most crystals being almost uniaxial. K-feldspar optics are rather susceptible to heating, but whether this would correlate with any of the thermal events affecting the gneisses is impossible to establish on the available evidence.

\section{BASEMENT CALCRETE}

The first basement rocks encountered-in the bottom of core catcher 15-had the superficial appearance of a calcareous sandstone in smear slides and in thin section. However, in thin section the quartz grains were unusually angular and the micas preserved a definite fabric. Similar rocks appeared in the uppermost part of Core 16 , but merged rapidly downwards into undoubted basement gneisses.

To monitor the extent and profile of this calcareous zone in the core, small samples were detached at regular intervals from the rocks in core catcher 15 through Section 1 of Core 16. After crushing to a fine powder, they were analyzed for $\mathrm{CaCO}_{3}$ onboard Glomar Challenger using the rapid "carbonate bomb" technique. The results of the shipboard study are outlined in Figure 9. While the carbonate bomb method produces results no more precise than $\pm 1 \%$, and of accuracy somewhat worse than this, particularly at low carbonate levels, the results demonstrate the marked increase in carbonate content of the gneisses at the top of the old basement land surface, reaching a peak of $40 \% \mathrm{CaCO}_{3}$ at the Core $16 /$ core catcher 15 boundary. The apparent reduction in $\mathrm{CaCO}_{3}$ content at the actual surface $(15, \mathrm{CC}, 2)$ is probably genuine, since the poorly indurated sand immediately overlying the basement is virtually calcitefree. There is a fairly sharp fall in carbonate content between Samples $16(\# 1)$ and $16(\# 2)$, and the remaining part of Core 16 is characterized by carbonate contents of $10 \%$ or less. This represents the extensive calcite veining in the upper part of Core 16, Section 1 together with the replacement of plagioclase by carbonate. The scatter in the results is probably real, the more 


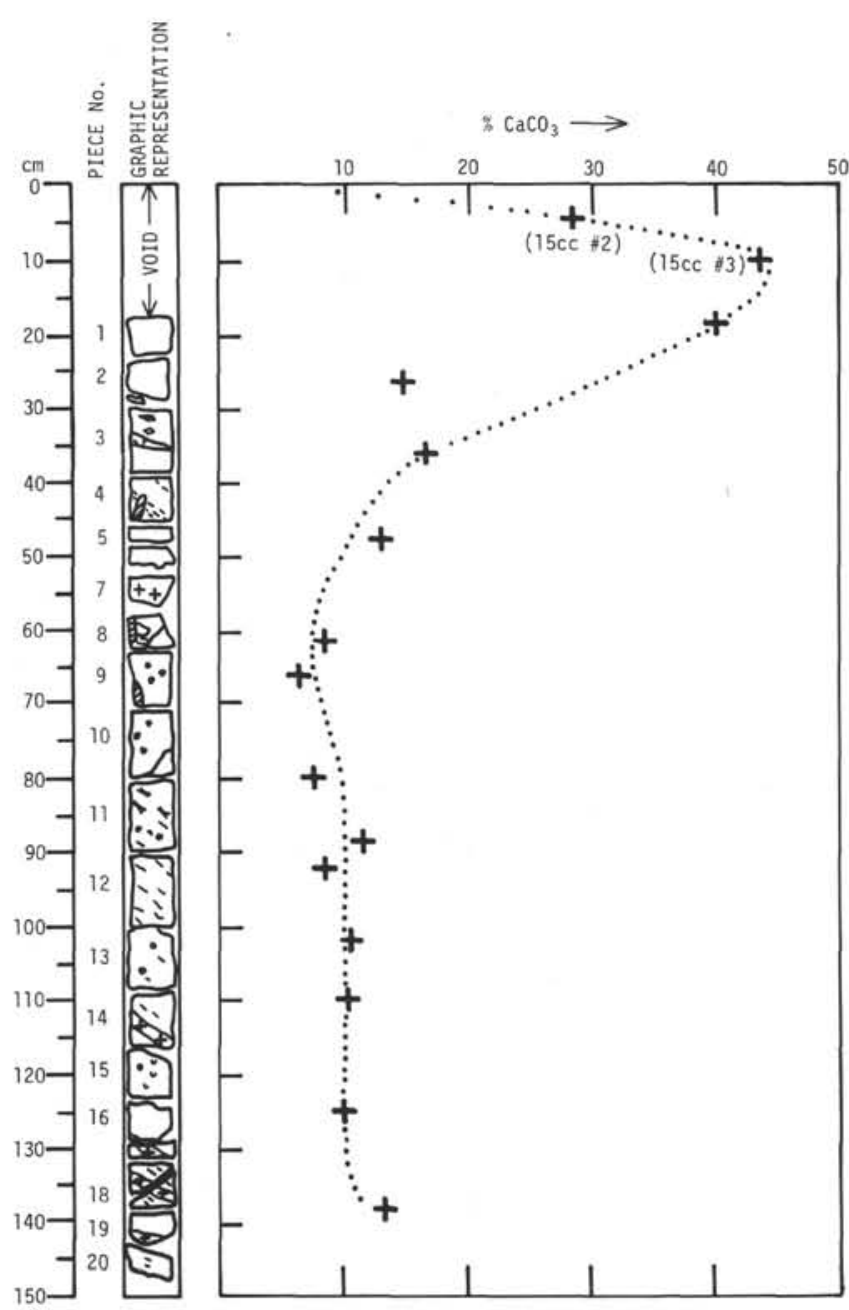

Figure 9. Distribution of calcite in the upper section of Core 16, Site 330. Samples from core-catcher 16, representing the basement surface, are included and are placed in their likely position relative to Core 16. Determination of calcite was carried out by the "carbonatebomb" method onboard ship.

plagioclase-rich gneisses recording the highest percentage of calcite, while the $\mathrm{K}$-feldspar pegmatite veins record the least.

Calcite-filled veins are present throughout the entire basement section, but are more abundant in the upper part of the core. The majority are vertically oriented with respect to the core. Within the rocks themselves plagioclase is the most easily replaceable phase, the plagioclases in the gneisses being more easily replaced than those in the pegmatitic veins, presumably because of the more calcic composition of the former ( $\mathrm{An}_{35}$ as against $\mathrm{An}_{20}$ ). Most of the plagioclases in the gneisses are clouded badly and are hardly recognizable from their optics. Quite low down in the core, the centers of the plagioclases may be replaced by fine-grained to well-crystallized calcite, or by well-crystallized siderite. In the upper half of Core 16, Section 1, the plagioclases are completely replaced by carbonate. The K-feldspars in the gneisses are invariably clouded, but otherwise are fairly resistant to replacement by calcite except locally in the carbonate-rich zone. The K-feldspar in the pegmatite veins is little affected, although there is evidence of recrystallization to finer grained, more highly birefringent feldspar adjacent to calcite-filled veins. There is no petrographic evidence that the other major minerals-quartz, biotite, chlorite, garnet, or tourmaline-are affected by carbonate replacement.

In the calcareous zone at the top of the basement core, the high carbonate content seems to have been accomplished by several processes:

1) Replacement of all the plagioclases in the gneisses by either fine-grained or well-crystallized calcite. Any $\mathrm{K}$-feldspar in these gneisses seems to have suffered the same fate, although some clouded degraded remnants are recognizable. The $\mathrm{K}$-feldspar pegmatite veins in this zone are much less affected.

2) In-filling of calcite between grain boundaries (Plate 4, Figures 3, 4). This results in the pulling-apart of quartz crystals and biotite aggregates, although the original fabric of the rock is preserved intact, or only slightly disturbed. The crystallinity and color of the biotites is unaffected: brown and green biotites remain interlayered as in the gneisses lower down the core.

3) Partial solution of the quartz grains, resulting in irregular boundaries. It is difficult, however, to assess reliably the extent of this: the quartz content of the carbonated gneisses is still high. Also, in the uppermost calcareous zone there appears to be some evidence of the biotites being replaced by irregular crystals and aggregates of pale green fine-grained chlorite.

The resulting heavily carbonated basement consists largely of angular quartzes and biotite aggregates in a matrix of up to $40 \%$ calcite. The calcite is either a mixture of fine- to medium-grained crystals of random orientation (Plate 4, Figure 4), or (as in 16-1, \#3) large well-crystallized grains up to $1 \mathrm{~cm}$ in diameter (Plate 4, Figure 3). These large poikilitic calcites may almost totally enclose aggregates of quartz and biotite, but the original fabric is still preserved. Extensive though this carbonation is, the original pegmatitic veins are still recognizable, even in the topmost sample: in these both the sodic plagioclase and the $\mathrm{K}$-feldspar are only partially replaced by calcite.

A few aggregates of small laths of colorless gypsum are present in the uppermost heavily calcitized basement. Pyrite is also a frequent, but trace, constituent in this calcreted zone, but it seems to lie along planes following original calcite veins. Such veins, with occasional small pyrites, are found in the upper part of Core 16, Section 1. Detrital pyrites, forming delightful "hopper" octahedra, are common in the sandy horizon immediately overlying the basement, but whether the two have a common origin is debatable: no "hopper" octahedra were noted in the basement veins.

The petrographic features detailed above suggest that the calcareous-rich capping to the basement has developed by a process of veining and replacement of unstable minerals such as plagioclase, followed by growth of calcite along intergrain boundaries and eventual leverage of the grains apart, perhaps assisted in the later stages by slight dissolution of the silicate minerals. The fact that the fabric of the rock is left essentially undisturbed indicates that the process was a gradual one, perhaps operative over a period of many 
thousands of years. This is supported by the large size of some of the poikilitic calcites. The presence of an unconsolidated sand immediately overlying the basement suggests that the process is unconnected with the sedimentation which eventually buried the basement, but instead is more directly related to the subaerial profile of the old basement land surface.

The most logical explanation is that the calcareous zone represents a palaeobasement calcrete or caliche, a widespread surface phenomenon in many parts of the world. The formation and significance of this basement calcrete is discussed further below.

\section{Geochemical Effects Associated with Calcrete Formation}

The core driven through the basement of Hole 330 provides an unusual opportunity to test the geochemical effects on bedrock of calcrete formation: albeit probably the last place on earth to conceive of such a study! Some difficulties arise, of course, due to the variable lithology and chemistry of the bedrock itself. Nevertheless, even allowing for this, there are certain obvious chemical changes which can be directly correlated with depth in the core. The most significant elements which vary systematically are shown in Figure 10. The remaining variations are apparent from the analyses listed in Table 2.

As might be expected, both calcium and carbondioxide show a marked increase near the top of Core 16 , Section 1. Calcium itself barely exceeds $1 \%$ by weight through most of Cores 17 and 16, Section 2, but reaches $9.55 \%$ at the top of Core 16 . Carbon dioxide is similarly low to only just detectable in the lower parts of Core 17, but reaches $6 \%$ at the top of Core 16. The more detailed shipboard study of the abundance and distribution of calcite in the calcrete zone of Core 16 is shown in Figure 9, and demonstrates the restriction of abundant calcrete to the upper few inches of Core 16 (and 15, CC).

Whether there is a complementary actual decrease in the abundance of calcium in the rocks lower down in the core is not too clear because of the general scatter of values, but this obviously bears upon the origin of the calcrete. Petrographic examination shows clearly that

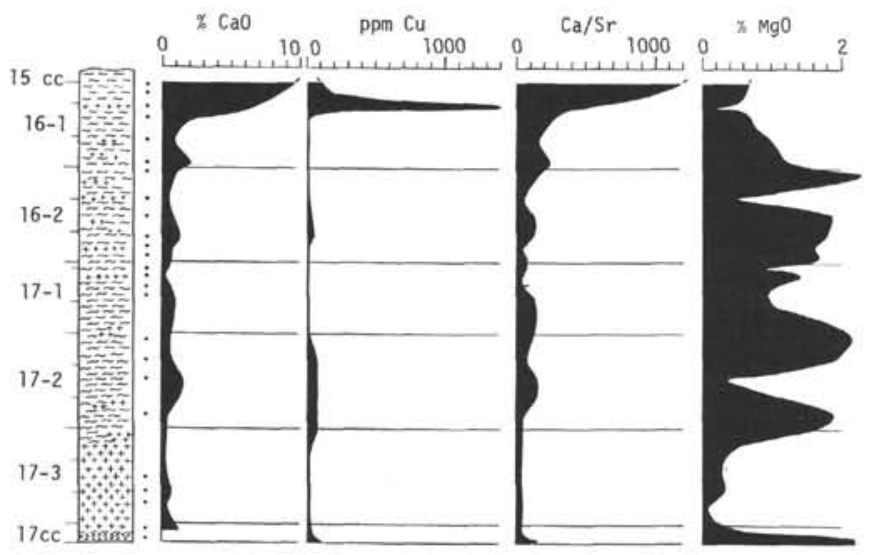

Figure 10. Depth-profiles of element and element ratio variation related to calcrete formation in the cored basement at Site 330. most of the plagioclases in the gneisses throughout the core are partially to wholly replaced by carbonate, although the more sodic plagioclases are less affected. The proportion of plagioclase is of course low in the gneisses, but it is possible that breakdown of plagioclase throughout a greater depth of bedrock could free the necessary amount of calcium to form the calcrete. It is equally possible that most of the calcite was ultimately derived from soluble bicarbonate in the circulating ground water.

Significant changes in the other major elements are more difficult to decipher due to the general scatter. A reduction of up to $20 \%$ might be expected in the calcrete zone itself in order to account for the increase in the abundance of calcite. However the concentration of magnesium in the calcrete zone is barely half that in the remainder of the core (granitic rocks excluded). This indicates that dolomite has not been precipitated along with the calcite. The levels of phosphorus in the calcrete zone are, however, less than a tenth that of the mean value in the lower part of the core. The reason for this is difficult to understand. Phosphorus is generally concentrated in apatite, where calcium is also an essential component, but apatite is a common detrital mineral which generally survives the weathering cycle. Other elements which might be regarded as fairly soluble, such as the alkalies, have a lower concentration in the calcrete zone, but not especially so.

Perhaps the most significant elemental variation obviously related to calcrete formation is that of copper. Values in the calcrete zone itself are high, reaching 1400 ppm in Section 330-16-1, (\#7). Yet but a few centimeters below this, in Section 330-16-1, (\#10) copper is barely detectable. Similar low values persist until well down into Core 17, where what might be regarded as normal values of around $100 \mathrm{ppm}$ occur. The granite pegmatite itself is deficient in copper, yet the equivalent vein in the calcrete zone has $1400 \mathrm{ppm}$. The relationship observed corresponds well with the well-known phenomenon of ground water leaching and secondary enrichment in areas of copper sulfide mineralization.

One might have expected other chalcophile elements such as zinc, nickel, and lead to behave similarly. However, there is no comparable variation in zinc concentration-the values in the calcrete zone are, if anything, rather smaller than in the remainder of the core. This implies that zinc is not present as a sulfide in any of the basement rocks. Similar remarks apply to nickel. Lead does, however, show a comparable variation to copper, but the relationship is not so obvious due to the duality in the geochemical behavior of lead: as a chalcophile element in galena, and as a substitute for potassium in $\mathrm{K}$-feldspar, where its ionic radius is similar. Variations in lead abundance are best logged as the $\mathrm{K} / \mathrm{Pb}$ ratio (normally $\sim 1000$ in most rocks). This ratio is indeed about 1000 throughout most of Core 17, but is approximately twice this value in the gneisses of Core 16, suggesting that lead has been leached to some extent along with copper. There is, however, no comparable zone of enrichment in the calcrete.

The other trace elements show no obvious variation, apart from the expected small reduction in the calcrete zone itself. Curiously, some complementary variation 
between strontium and calcium might have been expected due to the common sympathetic behavior between these two elements. However, it is clear from the analyses that a large proportion of the strontium present in the rocks must lie in K-feldspar, which in fact is one of the least-altered minerals in the basement rocks. The strontium in the calcrete zone is therefore essentially that in the original rocks: there has been no precipitation of strontium carbonate along with calcium carbonate. This is evident from the marked increase in $\mathrm{Ca} / \mathrm{Sr}$ ratio in the upper part of Core 16 .

\section{Climatic Significance of Calcrete}

The term "calcrete" was first widely introduced by Lamplugh in 1902 to describe the calcareous duricrusts arising through secondary near-surface precipitation of calcium carbonate in soils, subsoils, superficial deposits, or bedrock in many parts of the world. Alternative terms in common usage include "caliche" (N. America), "kankar" (India), tufa (S. Africa), croute calcaire (France), and many others. Other types of in situ duricrust are also known: silica (silcrete) and laterite (alcrete or ferricrete).

The global distribution of calcrete has been discussed by Goudie (1973). The best-developed calcretes in the world occur in semi-arid regions such as the High Plains of the U.S.A., the Pampas of Argentina and the coastal lowlands of Venezuela, the Kalahari of southern Africa and other parts of East and North Africa, parts of southern France and Spain, and equivalent arid areas in India, Australia, and the USSR.

While a climatic dependence of calcrete is obvious, it is not always possible to define the climatic conditions unambiguously due to the fact that many calcretes are paleo rather than recent, and may have evolved under different climatic conditions than those pertaining at present. Major climatic variations, at least in certain regions, are known even within historic times. Nevertheless, there is an obvious dependence of calcrete formation on mean annual rainfall, mean annual temperature, and hence on the rate of evapotranspiration. Other variables include the distribution of rainfall throughout the year, vegetation, and the source (whatever it is) of the carbonate itself. In N.E. Australia, Watkins (1967) has investigated the distribution of calcrete in relation to climate and has shown that calcrete borders the arid regions, and he suggests that calcrete may in turn be bordered by silcrete and eventually by laterite in progressively more humid zones. There is an obvious balance between rainfall and mean temperature: calcretes can form under more humid conditions, given a higher mean annual temperature. However, from the compilation provided by Goudie (1973), a humid limit of $\sim 500 \mathrm{~mm}$ annual precipitation is common for regions where the mean annual temperature is on the order of $18^{\circ} \mathrm{C}$. A dry limit is much more difficult to define, but calcrete does not appear to be common in areas with less than 100-200 $\mathrm{mm}$ annual precipitation. On the other hand, concentration of the rainfall into a peaked summer season where precipitation exceeds evapotranspiration tends to discourage calcrete formation by allowing accumulated calcium carbonate to be leached out of the calcrete zone into surface waters. In spite of these exceptions and provisos, there is on a global scale a good correlation between high-calcrete areas and climate.

The occurrence of calcreted basement at Site 330 may therefore set some climatic limitation on this sector of Gondwanaland during the Early to Mid-Jurassic. The above discussion would suggest a climate similar to the modern Mediterranean or North Africa. This must be set against the knowledge of an early Permian glaciation in this sector of Gondwanaland on the one hand, and the widespread amelioration of climate during the Cretaceous on the other. Swett's (1974) discovery of calcrete in the Arctic gives a slight flexibility to interpretations here!

\section{Origin of the Basement Calcrete at Site $\mathbf{3 3 0}$}

Many of the world's best-developed calcrete duricrusts appear to be Pliocene to early Pleistocene in age rather than recent (cf. Goudie, 1973). They may have developed gradually over a period of many millions of years. The mineral textures in the Hole 330 basement calcrete would seem to accord with this. While many calcretes have formed over terrains which are dominantly made up of calcareous bedrock, there are many examples of calcrete overlying such diverse rock types as basalt, granite, gneiss, schist, shales, or mudstone (Goudie, 1973). In such cases the source of the carbonate poses a problem: has it been derived by deep weathering of calcic minerals such as plagioclase, augite, hornblende, garnet, etc, in the underlying bedrock, or has the carbonate been introduced as soluble calcium bicarbonate in "hard" ground waters possibly from a distant calcareous source? Certainly ground waters in areas of calcrete formation have calcium contents in the range of $30-200 \mathrm{ppm}$ (Goudie, 1973), but also have concentrations of magnesium and sodium of similar magnitude, which are not represented by equivalent mineral phases such as dolomite or halite. In the Hole 330 calcrete, too, the only additional secondary phase apart from calcite is the trace amount of gypsum, another calcic mineral.

Although hypotheses of a ground water origin for calcrete have been in vogue for many decades, difficulties such as the absence of other mineral phases, or varying depths to the water table, and doubts as to whether capillary rise of ground water is possible over depths greater than a few meters, have led to alternative suggestions. Those applicable to the Hole 330 situation would be (a) deep weathering by downward percolating rainwater to the extent of breakdown of calcic phases such as plagioclase followed by capillary rise and nearsurface precipitation of calcite during the dry season, or (b) addition of calcium to the land surface as aeolian or loess dust or by rainwater itself (ca. $6 \mathrm{ppm} \mathrm{Ca}$ ) followed by solution weathering and reconcentration in the calcrete zone.

Data from the Hole 330 basement do not allow us to discriminate between any of these hypotheses with confidence. Certainly decomposition of the gneiss plagioclases could have provided the necessary calcium if ac- 
companied by a simultaneous lowering of the land surface by the normal processes of erosion. In this case the other elements released $(\mathrm{Na}, \mathrm{Sr})$ may never have reached supersaturation level in the calcrete zone and may have been flushed away by the next rainfall. Whether the plagioclase did provide the calcium, however, is not clear, since most of the plagioclases in the core are partially replaced by calcite and therefore record a net gain rather than loss of calcium. Likewise ground water could have provided the calcite-rich zone by capillary action if the annual rainfall was high enough to remove the more soluble constituents $(\mathrm{Na}, \mathrm{K}, \mathrm{Sr}, \mathrm{Mg})$ temporarily precipitated in the calcrete zone. This mechanism would accord better with the replacement of plagioclases lower down the core by calcite. There is no positive evidence in favor of aeolian or loess dust as the source for the calcrete.

\section{SUMMARY: GEOLOGICAL HISTORY OF THE FALKLAND BASEMENT}

Taking a 19.5-meter linear core sample stab into a section of basement metamorphics with $3 \mathrm{D}$ complexity over an area of perhaps thousands of square kilometers is equivalent to taking a 1-lb grab-sample of lunar regolith in the hope of elucidating the evolutionary history of the moon. With faith a consistent story may emerge-but one which is likely to be very susceptible to modification with the acquisition of further data. As the gathering of further data on the remaining basement of the Falkland Plateau may be subject to considerable delay, it seems opportune to consolidate the available data in terms of the likely geological history of the region in the hope that worthwhile comparisons may eventually be made with continental segments which initially lay adjacent to the Falkland Plateau. It goes without saying that these isolated continental rocks could hardly have developed as they have without being part of a much larger continental mass. Thus they are proof, if any still be needed, of the reality of continental drift.

The specific points of interest are as follows:

1) The gneisses represent interbanded mature sediments varying in composition from pelite through semipelite to psammite, and possibly with subsidiary arkose. There are no rock types directly corresponding to graywacke. The geochemical and isotopic evidence would be consistent with derivation of the sediments from an older Precambrian granitic terrain. While the sediments themselves could well be Precambrian in age, they are compositionally different from most Archaean gneisses which seem to be of plutonic origin.

2) The sediments were metamorphosed at high grade to develop the primary assemblages characterized by red-brown biotite, garnet, and orthoclase. Textural relationships suggest, however, that the gneisses had a pre-existing coarser-grained fabric-perhaps attributable to a previous episode of metamorphism. The $\pi_{1}$ pegmatitic veins and quartz veins may be linked with this earlier phase of metamorphism, but were deformed and recrystallized during the second high-grade metamorphism. Small rounded garnets grew in both the gneisses and the $\pi_{1}$ pegmatites during this second metamorphic phase. Metasomatic effects connected with either or both of these metamorphic episodes caused some redistribution of potash within the gneisses and resulted in the smoothing out of extreme per-aluminous compositions which may account for the absence of alumino-silicate index minerals such as cordierite, kyanite, or sillimanite in the high-grade gneisses. The growth of tourmaline in the gneisses may also be connected with such a metasomatic episode. Moreover, the abundance of tourmaline in the residual sand overlying the basement suggests that tourmalinization is regional. The present even-grained granular texture of the more psammitic gneisses is almost certainly linked with the phase of high-grade metamorphism.

3) A major phase of injection of granite pegmatite $\left(\pi_{2}\right)$ followed the high-grade metamorphism. Geochemical and isotopic data would allow derivation of the pegmatite by anatexis of the gneisses at greater depths. Large vermicular garnets grew in the margin of the pegmatite and in the adjacent semipelitic gneisses shortly following the emplacement of the pegmatite. The granite pegmatite and associated veins were not however deformed. Synchronous with the emplacement of the pegmatite, and probably also predating and postdating its emplacement, was a series of retrogressive effects. The most significant caused replacement of much of the reddish-brown biotite by green hydrobiotite with much lower $\mathrm{Ti}$ and $\mathrm{K}$ content (transitional toward chlorite). The early garnets were partly replaced by chlorite, as were the biotites in the granite pegmatite. Retrogression seems to have continued to a much later stage and at lower grade in the form of fine sericite/pyrophyllite veins which traverse the gneisses.

These retrogressive effects, which clearly brought about removal of potassium from the main potassic phase, biotite, would almost certainly have produced similar changes in the contents of $R b$ and accumulated radiogenic $\mathrm{Sr}$. It may well be that the $\mathrm{Rb}-\mathrm{Sr}$ isochron age of 535 m.y. for the gneisses correlates with this event, in which case the need to accommodate one or more episodes of metamorphism and deformation into the chronology might extend the age of deposition of the sediments well back into the Precambrian. The retrogression seems to have been more of a hydrothermal than a prolonged thermal event, however, since the $\mathrm{K}$-feldspars in both the gneisses and the pegmatites show only slight evidence of inversion to microcline.

4) Intrusion of basic veins and a microsyenite followed the emplacement of the $\pi_{2}$ pegmatite. Both have fine-grained textures suggesting intrusion into cooler host rocks, but the fact that they are altered would indicate that they may have been emplaced before the cessation of the hydrothermal activity.

5) Some faulting seems to have affected the rocks at a fairly late stage in their history. This is manifest in small fault hitches (with a throw of a few centimeters) between contrasting rock types at several places in the core, and with more severe fracturing and brecciation near the contact between the granite pegmatite and the gneisses. The faulting could be connected with uplift of the gneisses.

6) Considerable erosion must have taken place to expose the deeply buried gneisses. The later stages of this denudation (in the Early Jurassic?) seem to have taken 
place under rather warm, semi-arid and subaerial conditions, thus allowing the development of a calciteimpregnated zone (calcrete) in the upper few tens of centimeters of the old land surface. The calcium necessary for the calcrete development was probably derived from the eroded rocks and progressively concentrated by the leaching/capillary action of ground water. Eventual burial of the irregular land surface under lagoonal Jurassic sediments preserved the relationships before the Falkland continental segment subsided to its present depth $3 \mathrm{~km}$ below the South Atlantic.

It is possible that the cycle of rapid erosion and uplift of the gneissic basement followed by equally rapid subsidence and burial reflects the ridge activity associated with the opening of the South Atlantic. On the other hand, this may be no more than coincidence-most small foundered continental blocks appear to have subsided below sea level.

\section{Regional Comparisons}

The significance of the ages of the Site 330 basement rocks within the context of a reconstructed Gondwanaland is discussed fully in the companion paper by Beckinsale et al. (this volume). More specifically, the nearest outcrop of comparable basement occurs at the western end of the Falkland Plateau, forming a $5-\mathrm{km}$ strip along the base of the cliff at Cape Meredith, West Falkland Island. The basement rocks were described briefly by Baker (1924) in his report on the geology of the Falkland Islands. More recently Dr. C.G. Smith of the British Antarctic Survey made a further brief visit to the area in 1969.

The rocks of the Cape Meredith Complex are overlain unconformably by gently dipping strata of the Lower Devonian Port Stephens Beds. Baker (1924) referred to them as "Archaean." His brief descriptions together with the observations of Smith (personal communication) suggest that the whole complex has many similarities with the Site 330 basement. The metasedimentary component of the complex is represented by quartzites and dark semipelitic schists composed largely of quartz and green "hornblende" (? green hydrobiotite). There are in addition augen gneisses (composed of $\mathrm{K}$-feldspar, quartz, and biotite); coarse pink granites and granite pegmatites (with K-feldspar, quartz, garnet, and biotite); numerous basic dykes and also lamprophyric dykes (with olivine, augite, hornblende, and $\mathrm{K}$-feldspar).

Judging from these descriptions, it would appear that most of the components of the Site 330 basement are present at Cape Meredith, and that the relationships between the various rock types may be similar. This remarkably close similarity between the eastern and western extremities of the Falkland Plateau basement might suggest that the rest of the plateau is made up of similar rock types and has undergone a similar geological history.

\section{ACKNOWLEDGMENTS}

I would like to thank G.L. Hendry and N. Donnellan for assisting in the geochemical analysis of the samples and R.N. Wilson for carrying out the microprobe determinations. I am grateful to F. Moseley for valuable discussions on calcrete formation and to A.E. Wright for his constructive criticism of the manuscript.

\section{REFERENCES}

Baker, H.A., 1924. Final report on geological investigations in the Falkland Islands, 1920-1922: Stanley (Government Printer).

Condie, K.C., 1967. Geochemistry of early Precambrian graywackes from Wyoming: Geochim. Cosmochim. Acta, v. 31, p. $2135-2149$.

Deer, W.A., Howie, R.A., and Zussman, J., 1962. Rockforming minerals. v. 1. Ortho- and ring silicates: London (Longmans).

Engel, A.E.J. and Engel, C.G., 1960. Progressive metamorphism of the major paragneiss, northwest Adirondack Mountains, New York. Part II: Mineralogy: Geol. Soc. Am. Bull., v. 71, p. 1-57.

Fitton, J.G. and Gill, R.C.O., 1970. The oxidation of ferrous iron in rocks during mechanical grinding: Geochim. Cosmochim. Acta, v. 34 , p. $518-524$.

Goudie, A., 1973. Duricrusts in tropical and subtropical landscapes: Oxford (Clarendon Press).

Heier, K.S., 1973. Geochemistry of granulite facies rocks and problems of their origin: Phil. Trans. Roy. Soc. London, Ser. A, v. 273 , p. $429-442$.

Holland, J.G. and Lambert, R. St. J., 1975. The chemistry and origin of the Lewisian gneisses of the Scottish Mainland: the Scourie and Inver assemblages and subcrustal accretion: Precambrian Res., v. 2, p. 161-188.

Jeans, P.F., 1973. Unpublished Ph.D. Thesis, University of Birmingham.

Lambert, I.B. and Heier, K.S., 1968. Geochemical investigations of deep-seated rocks in the Australian Shield: Lithos, v. 1, p. 30-53.

Lamplugh, G.W., 1902. Calcrete: Geol. Mag., v. 9, p. 75.

Leake, B.E., Hendry, G.L., Kempe, A., Plant, A.G., Harvey, P.K., Wilson, J.R., Coats, J.S., Aucott, J.W., Lunel, T., and Howarth, R.J., 1969. The chemical analysis of rock powders by automatic X-ray fluorescence: Chem. Geol., v. 5 , p. $7-86$.

Moorbath, S., 1975. Evolution of Precambrian crust from strontium isotopic evidence: Nature, London, v. 254, p. $395-398$.

Parry, G., 1973. Unpublished Ph.D. Thesis, University of Birmingham.

Pettijohn, F.J., 1963. Chemical composition of sandstones-excluding carbonate and volcanic sands. Data of Geochemistry (6th ed.): U.S. Geol. Surv. Prof. Paper 440$\mathrm{S}$.

Riley, P.J., 1958. Simultaneous determination of water and carbon dioxide in rocks and minerals: Analyst, v. 83, p. $42-49$.

Shaw, D.M., Reilly, G.A., Muysson, J.R., Pattenden, G.E., and Campbell, F.E., 1967. An estimate of the chemical composition of the Canadian Precambrian Shield: Canadian J. Earth Sci., v. 5, p. 829-853.

Sheraton, J.W., 1970. The origin of the Lewisian gneisses of Northwest Scotland, with particular reference to the Drumbeg area, Sutherland: Earth Planet. Sci. Lett., v. 8, p. 301-310.

Sheraton, J.W., Skinner, A.C., and Tarney, J., 1973. The geochemistry of the Scourian gneisses of the Assynt district. In Park, R.G. and Tarney, J. (Eds), The early Precambrian of Scotland and related rocks of Greenland: University of Keele, p. 13-30. 
Spooner, C.H. and Fairbairn, H.W., 1970. Strontium $87 /$ strontium 86 initial ratios in pyroxene granulite terrains: J. Geophys. Res., v. 75, p. 6706-6713.

Stoakes, M.R., 1971. Unpublished M.Sc. Project Thesis, University of Birmingham.

Swett, K., 1974. Calcrete crusts in an Artic permafrost environment: Am. J. Sci., v. 274, p. 1059-1063.

Tarney, J., 1976. Geochemistry of Archaean high grade gneisses, with implications as to the origin and evolution of the Precambrian crust. In Windley, B.F. (Ed.), The early history of the earth: London (Wiley), p. 405-417.

Tarney, J., Skinner, A.C., and Sheraton, J.W., 1972. A geochemical comparison of major Archaean gneiss units from Northwest Scotland and East Greenland: Rept. 24th Int. Geol. Congr., Montreal, v. 1, p. 162-174.

Watkins, J.R., 1967. The relationship between climate and the development of land forms in the Cainozoic rocks of Queensland: J. Geol. Soc. Australia, v. 14, p. 153-168. 



\section{PLATE 1}

Figure 1 Sample 330-16-1, 108-117 cm. Psammitic metasedimentary gneiss with granular texture. Weak foliation dipping at ca. $30^{\circ}$. Concordant $\pi_{1}$ pegmatitic vein. Dark patches in pegmatite vein and in gneiss are chlorite segregations after garnet.

Figure 2 Sample 330-16-2, 3-13 cm. Interbanded biotitegarnet pelitic and granular psammitic gneiss. Concordant $\pi_{1}$ pegmatite vein. Lithological banding offset by small fault. Carbonate veins along fault plane.

Figure 3 Sample 330-16-2, 93-100 cm. Foliated semipelitic gneiss cut by slightly discordant $\pi_{2}$ coarse $\mathrm{K}$ feldspar pegmatite vein.

Figure 4 Sample 330-16-2, 63-73 cm. Interbanded granular psammitic gneiss and garnet-biotite pelite with strong foliation. Concordant quartz vein. 


\section{PLATE 1}
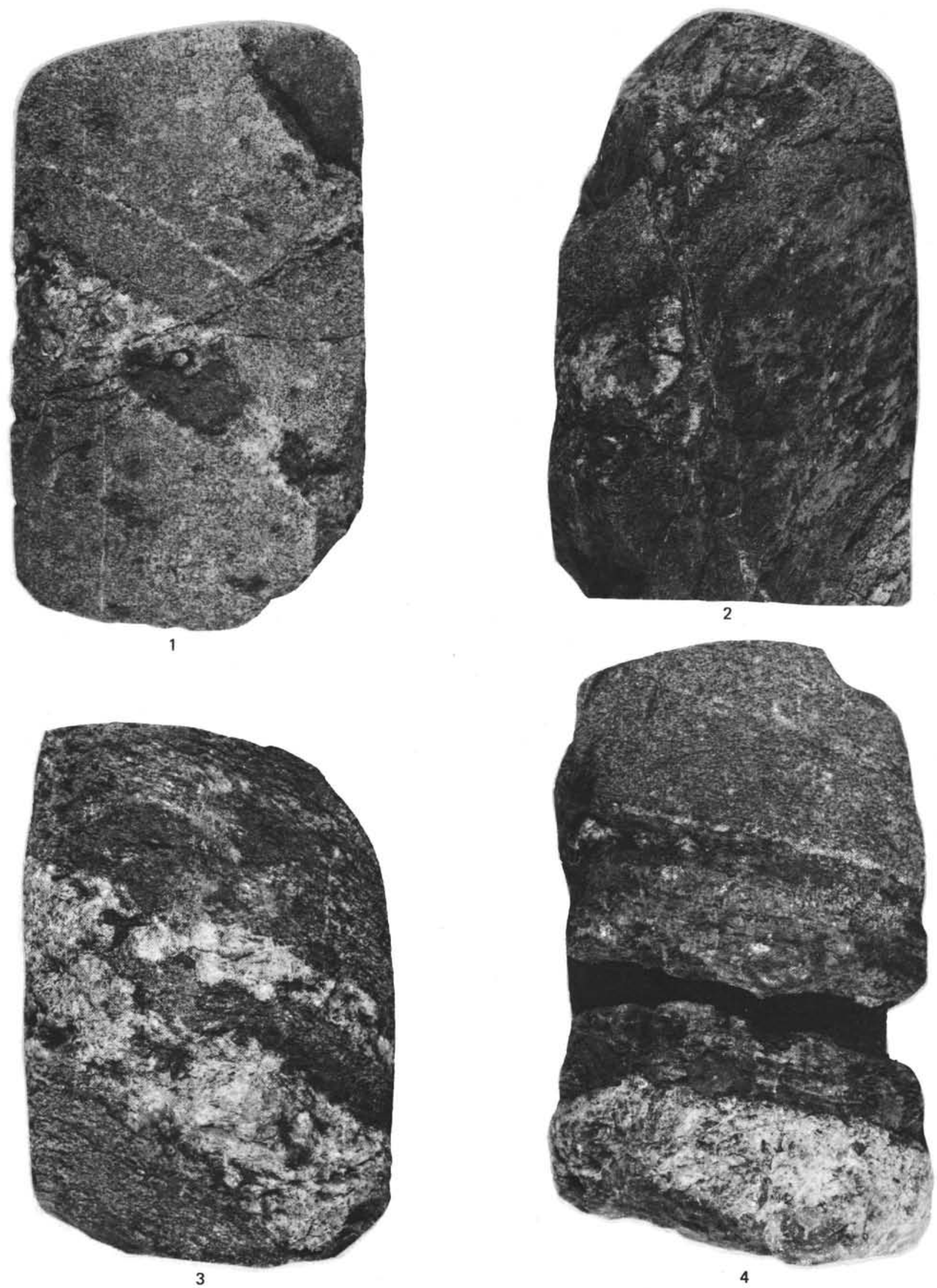


\section{PLATE 2}

Figure 1 Sample 330-16-2, 45-50 cm. Early $\pi_{1}$ K-feldspar pegmatitic vein with weak foliation. Dark patches represent garnets partly replaced by dark green chlorite.

Figure 2 Sample 330-17-3, 109-120 cm. Part of thick intrusive granite pegmatite $\left(\pi_{2}\right)$.

Figure 3 Sample 330-16-1, 39-46 cm. Thin basic vein cutting weakly foliated granular psammitic gneiss. Vein mainly dark green smectite clay with chlorite.

Figure 4 Sample 330-16-1, 58-64 cm. Basic vein cutting interbanded psammitic and semipelitic gneiss with slightly discordant pegmatite. Thin calcite veins, related to calcrete formation, occur along contact. 
PLATE 2
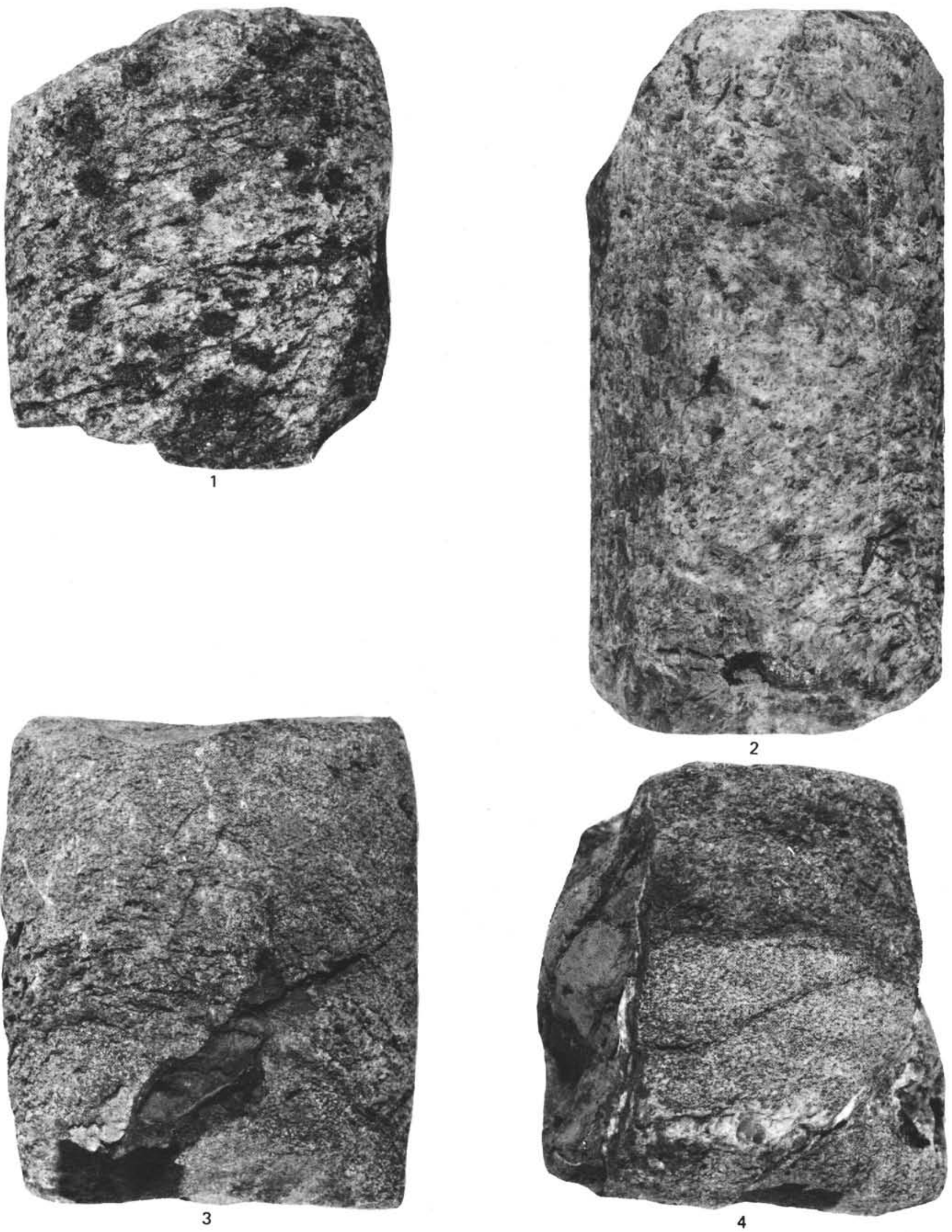


\section{PLATE 3}

Figure 1 Sample 330-16-2, \#1. Pelitic gneiss (biotitite). Large laths of brown biotite, largely replaced by green hydrobiotite with exsolution of titanomagnetite, aligned parallel to the foliation, $\times 25$.

Figure 2 Sample 330-16-2, \#21. Thin pelitic band in granular psammitic gneiss Foliation defined by biotite. $\times 25$.

Figure 3 Sample 330-17-1, \#3B. Semipelitic gneiss. Fabric in biotites parallel to central band, but biotites envelop polygonal quartzes. Fine-grained secondary sericite-filled veins on either side of central band. $\times 25$.

Figure 4 Sample 330-16-1, \#1 (crossed polars). Quartz-rich granulitic gneiss Note that quartz grains are elongated perpendicular to biotite fabric. $\times 25$.

Figure 5 Sample 330-16-2, \#18. Large tourmaline aligned parallel to foliation of semipelitic gneiss. $\times 25$.

Figure 6 Sample 330-16-2, \#5. Small rounded garnets in deformed $\pi$, K-feldspar pegmatite. Garnets partly replaced by fine-grained chlorite. $\times 25$. 


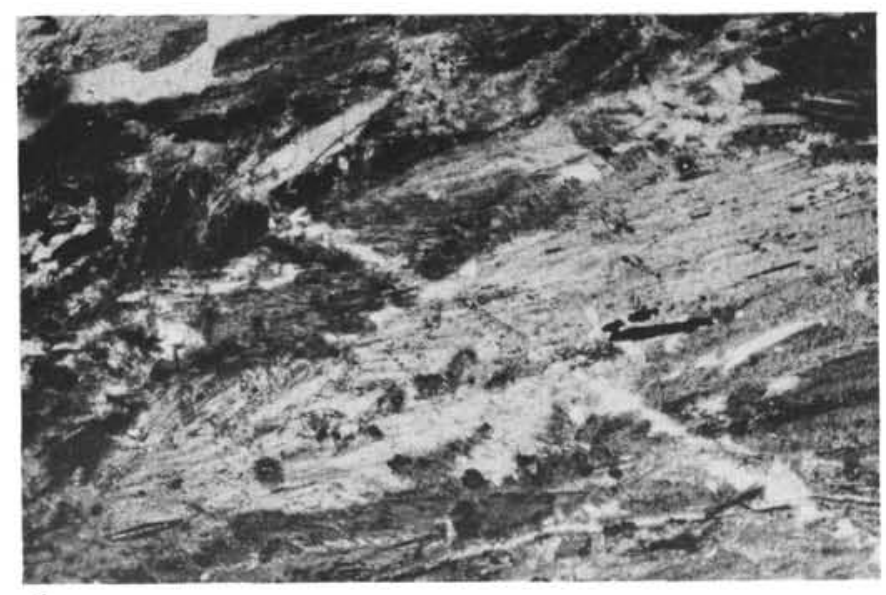

1

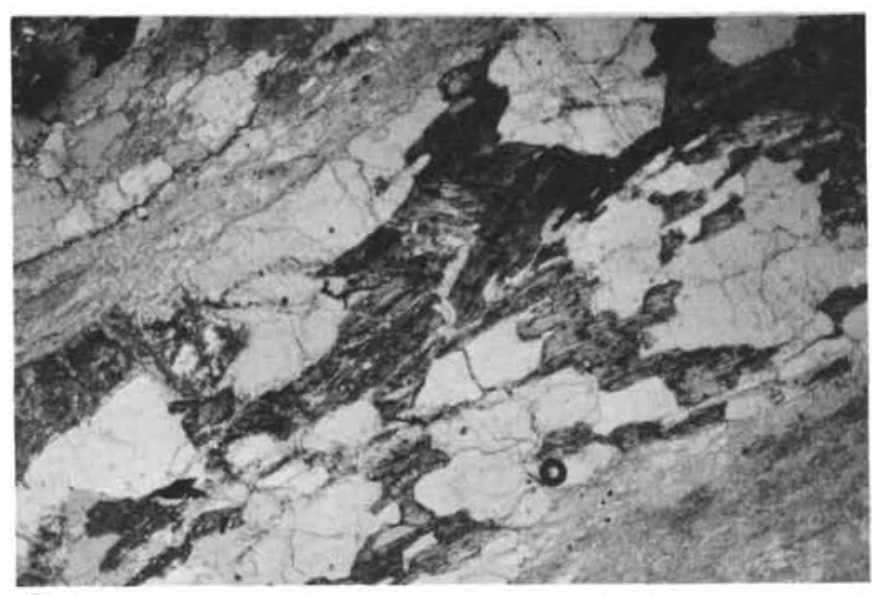
3
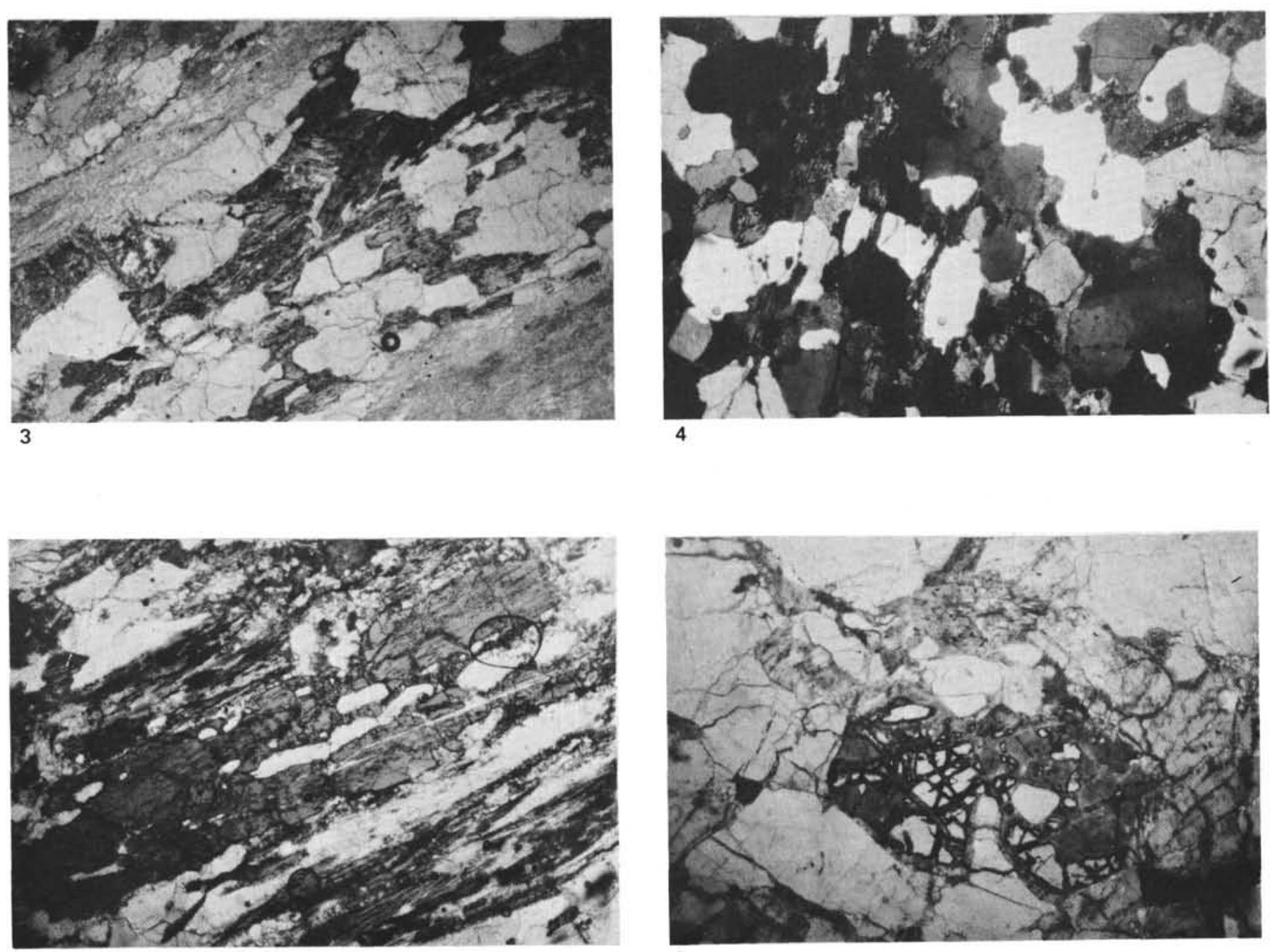

4

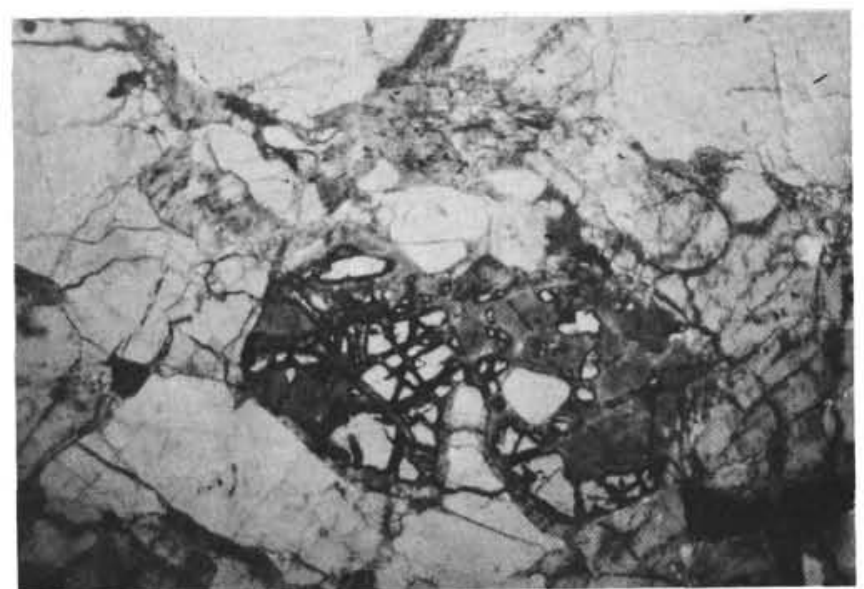

6 


\section{PLATE 4}

Figure 1 Sample 330-17-3, \#5. Garnet-quartz intergrowth at margin of granite pegmatite. $\times 25$.

Figure 2 Sample 330-17, CC, \#2. Intergrowth of quartz and $\mathrm{K}$-feldspar in granite pegmatite. $\times 25$.

Figure 3 Sample 330-16-1, \#3 (crossed polars). Calcitized granulitic quartz-rich gneiss. Quartzes, still in optical continuity, pulled apart and infilled by veins of calcite. Calcites in fact form 3 or 4 large poikilitic crystals within field of view.

Figure 4 Sample 330-16-1, \#1. Granulitic gneiss from calcrete zone. Quartzes pulled apart and infilled by fine-grained calcite. $\times 25$.

Figure $5 \quad$ Sample 330-17-2, \#3 (crossed polars). Plagioclase partly altered and replaced by fine-grained calcite. Alteration related to calcrete formation. $\times 25$.

Figure 6 Sample 330-17, CC, \#3. Vug filled with calcite and small quartz crystals in microsyenite. Mineralization related to late-stage alteration and calcrete formation. $\times 25$. 
PLATE 4
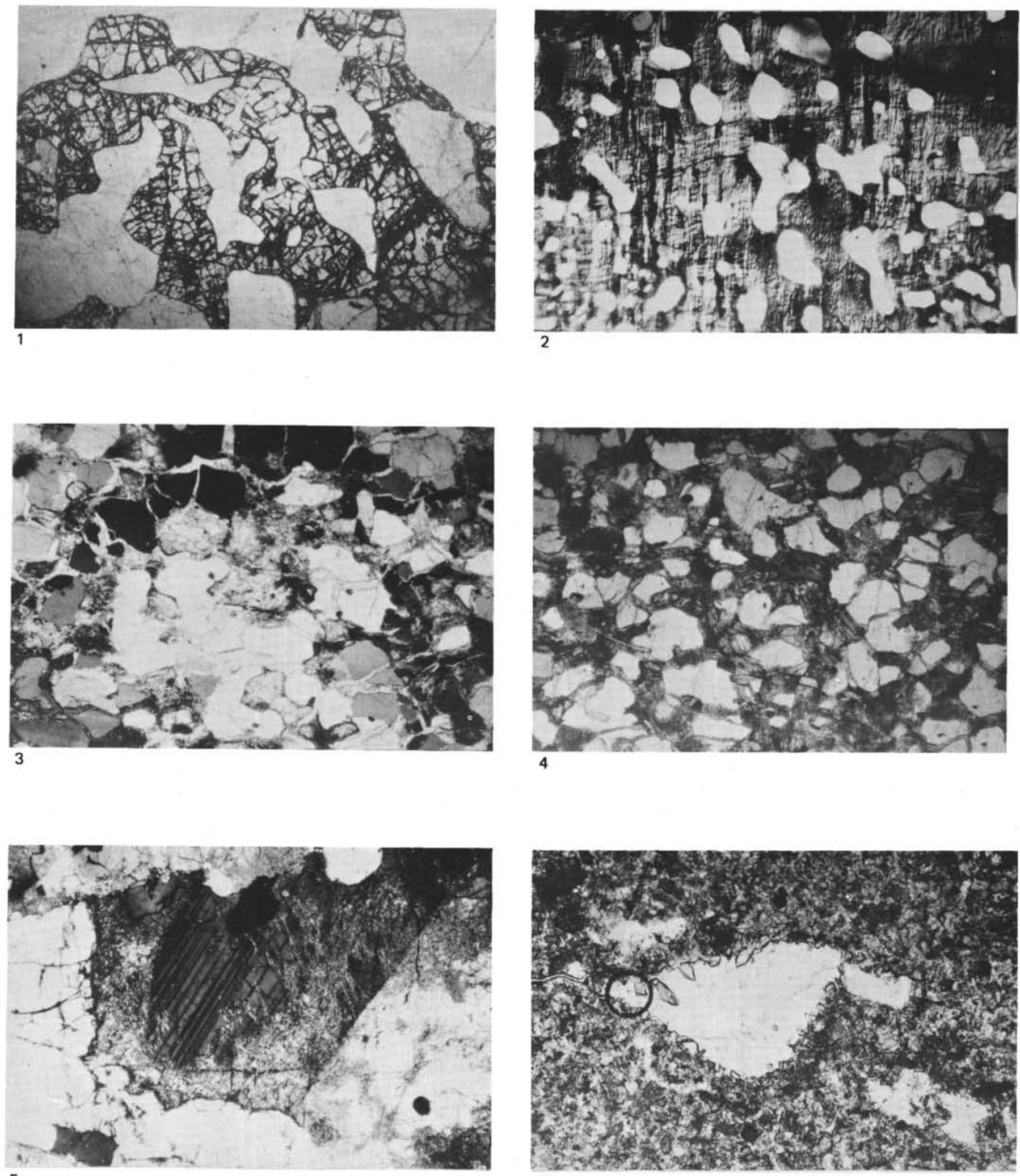

5 